\title{
Estimates on Green functions and Poisson kernels for symmetric stable processes
}

\author{
Zhen-Qing Chen ${ }^{1}$, Renming Song ${ }^{2}$ \\ 1 Department of Mathematics, Cornell University, Ithaca, NY 14853, USA \\ (e-mail: zchen@math.cornell.edu) \\ 2 Department of Mathematics, University of Illinois at Urbana-Champaign, 1409 W. Green St., \\ Urbana, IL 61801, USA (e-mail: rsong@math.uiuc.edu)
}

Received: 8 December 1997 / Revised version: 7 April 1998

Mathematics Subject Classification (1991): 60J30, 60J45, 60J75, 31C99

\section{Introduction}

One of the most basic and most important subfamily of Lévy processes is symmetric stable processes. A symmetric $\alpha$-stable process $X$ on $\mathbf{R}^{n}$ is a Lévy process whose transition density $p(t, x-y)$ relative to the Lebesgue measure is uniquely determined by its Fourier transform $\int_{\mathbf{R}^{n}} e^{i x \cdot \xi} p(t, x) d x=e^{-t|\xi|^{\alpha}}$. Here $\alpha$ must be in the interval $(0,2]$. When $\alpha=2$, we get a Brownian motion running with a time clock twice as fast as the standard one. Brownian motion plays a central role in modern probability theory and has numerous important applications in other scientific areas as well as in many other branches of mathematics. Thus it has been intensively studied. In this paper, symmetric stable processes are referred to the case when $0<\alpha<2$, unless otherwise specified. In the last few years there has been an explosive growth in the study of physical and economic systems that can be successfully modeled with the use of stable processes. Stable processes are now widely used in physics, operations research, queuing theory, mathematical finance and risk estimation. In some physics literatures, symmetric $\alpha$-stable processes are called Lévy flights, and they have been applied to a wide range of very complex physics issues, such as turbulent diffusion, vortex dynamics, anomalous diffusion in rotating flows, and molecular spectral fluctuations. In mathematical finance, stable processes can be used to model stock returns in incomplete market. For these and more applications of stable processes, please see the interesting book [14] by Janicki and Weron and the references therein and the recent article [15] by Klafter, Shlesinger and Zuomofen. In order to make precise predictions about natural phenomena and to better cope with these

Research of Z.-Q. Chen supported in part by an NSA grant. 
widespread applications, there is a need to study the fine properties of symmetric stable processes, just as for the Brownian motion case. Although a lot is known about symmetric stable processes and their potential theory (see [1, 2, 3, 4, 9, $10,12,14,16,17,20,21]$ and the references therein), little is known about the counterparts to some of the deep results for Brownian motion, such as sharp estimates on Green functions and Poisson kernels of bounded domains. In the special case of balls, the explicit formulae for the Poisson kernels and Green functions for symmetric stable processes are known. The formula for the Poisson kernel of balls were obtained by M. Riesz and the formula for Green function of balls were obtained by Blumenthal, Getoor and Ray in [3]. Prior to that, Kac, Polland, Spitzer, Widom, Kesten and Kinney had obtained some results for 1-dimensional case (cf. [3]).

Unlike the generator $\Delta$ of Brownian motion whose time clock is twice as fast as the standard one, the generator of a symmetric $\alpha$-stable process with $0<\alpha<2$ is the fractional Laplacian $-(-\Delta)^{\alpha / 2}$ which is non-local. Also a symmetric stable process is a process with discontinuous sample paths and having heavy tails, while Brownian motion has continuous sample paths and exponential decay tails. The transition density function $p(t, x-y)$ for discontinuous symmetric $\alpha$-stable process $X$ is approximately $c|x-y|^{-(n+\alpha)}$ when $|x-y|$ is large. So $X_{t}$ has infinite variance and when $0<\alpha \leq 1,\left|X_{t}\right|$ even has infinite mean. All these indicate the significant difference between Brownian motion and symmetric $\alpha$ stable processes.

In this paper, we will address some of these problems. More specifically, we will derive precise estimates on Green functions and Poisson kernels of $X$ in bounded $C^{1,1}$-smooth domains $D$. That $D$ is $C^{1,1}$ means that for every $z \in \partial D$, there exists a $r>0$ such that $B(z, r) \cap \partial D$ is the graph of a function whose first derivatives are Lipschitz. These estimates are very useful in studying other properties of symmetric stable processes. As examples of applications of these estimates, we prove that the $3 \mathrm{G}$ Theorem holds for symmetric $\alpha$-stable processes on bounded $C^{1,1}$ domains and that the conditional lifetimes for the symmetric $\alpha$-stable processes in a bounded $C^{1,1}$ domain are uniformly bounded.

To state the main results of this paper, let $X$ be a symmetric $\alpha$-stable process on $\mathbf{R}^{n}$ with $n \geq 2$ and $0<\alpha<2$. The process $X$ is transient and we are going to use $G$ to denote the potential of $X$. We know that the Green function of $X$ is given by

$$
G(x, y)=\int_{0}^{\infty} p(t, x, y) d y=2^{-\alpha} \pi^{-\frac{n}{2}} \Gamma\left(\frac{n-\alpha}{2}\right) \Gamma\left(\frac{\alpha}{2}\right)^{-1}|x-y|^{\alpha-n}
$$

(see, for example, [2]). Here $\Gamma$ is the Gamma function defined by $\Gamma(\lambda)=$ $\int_{0}^{\infty} t^{\lambda-1} e^{-t} d t$ for $\lambda>0$. For a domain $D$ in $\mathbf{R}^{n}$, let $\tau_{D}=\inf \left\{t>0: X_{t} \notin D\right\}$. Adjoin a cemetery point $\partial$ to $D$ and set

$$
X_{t}^{D}(\omega)= \begin{cases}X_{t}(\omega), & \text { if } t<\tau_{D} \\ \partial, & \text { if } t \geq \tau_{D}\end{cases}
$$


$X^{D}$ is called the subprocess of the symmetric $\alpha$-stable process $X$ killed upon leaving $D$, or simply the symmetric $\alpha$-stable process in $D$. It is well known that there is a continuous symmetric function $G_{D}(\cdot, \cdot)$ defined on $D \times D$ except along the diagonal such that for any Borel measurable function $f \geq 0$,

$$
E_{x}\left[\int_{0}^{\tau_{D}} f\left(X_{s}\right) d s\right]=\int_{D} G_{D}(x, y) f(y) d y .
$$

$G_{D}$ is called the Green function of $X$ in $D$. Note that $G_{D}$ has the following scaling property: for $a>0$,

$$
G_{D}(x, y)=a^{\alpha-n} G_{D / a}(x / a, y / a), \quad x, y \in D .
$$

The main results of of this paper are summarized as follows.

Theorem 1.1. Suppose that $D$ is a bounded $C^{1,1}$ domain in $\mathbf{R}^{n}$. Let $\delta(x)=$ $d(x, \partial D)$ be the Euclidean distance between $x$ and $\partial D$. Then there exists $a$ $C=C(D, \alpha)>0$ such that for $x, y \in D$,

$$
\begin{aligned}
G_{D}(x, y) & \leq C \frac{1}{|x-y|^{n-\alpha}}, \\
G_{D}(x, y) & \leq C \frac{\delta(x)^{\alpha / 2}}{|x-y|^{n-\alpha / 2}}, \\
G_{D}(x, y) & \leq C \frac{\delta(x)^{\alpha / 2} \delta(y)^{\alpha / 2}}{|x-y|^{n}}, \\
G_{D}(x, y) & \leq C \frac{\delta(x)^{\alpha / 2}}{\delta(y)^{\alpha / 2}} \frac{1}{|x-y|^{n-\alpha}} .
\end{aligned}
$$

Now that since $G_{D}$ is a symmetric function, so (1.6) tells that

$$
G_{D}(x, y) \leq C \frac{\delta(y)^{\alpha / 2}}{\delta(x)^{\alpha / 2}} \frac{1}{|x-y|^{n-\alpha}} .
$$

Theorem 1.1 is proved by using inversion with respect to spheres along with the explicit formulae for Green functions and exit distributions of $X$ on balls. For the now classical upper bounds for Green functions of Brownian motion, one can see Zhao [23] and the references therein. Comparing with the upper bound estimates above, the following lower bounds on the Green functions are much more difficult to prove. The proof involves some very detailed hard analysis. These lower bounds, in a sense, are generalizations of the results of Zhao [23] for Brownian motions to the discontinuous symmetric stable processes.

Theorem 1.2. Suppose that $D$ is a bounded $C^{1,1}$ domain in $\mathbf{R}^{n}$. Then there exists $a C=C(D, \alpha)>0$ such that for $x, y \in D$,

$$
\begin{aligned}
& G_{D}(x, y) \geq \frac{C}{|x-y|^{n-\alpha}}, \quad \text { if }|x-y| \leq \max \left\{\frac{\delta(x)}{2}, \frac{\delta(y)}{2}\right\} \\
& G_{D}(x, y) \geq C \frac{\delta(x)^{\alpha / 2} \delta(y)^{\alpha / 2}}{|x-y|^{n}}, \quad \text { if }|x-y|>\max \left\{\frac{\delta(x)}{2}, \frac{\delta(y)}{2}\right\} .
\end{aligned}
$$


The above lower and upper bounds provide precise information about the Green functions. Summarizing them up, we have

Corollary 1.3. Therefore there is a constant $C=C(D, \alpha)>1$ such that

$$
\begin{aligned}
& C^{-1} \min \left\{\frac{1}{|x-y|^{n-\alpha}}, \frac{\delta(x)^{\alpha / 2} \delta(y)^{\alpha / 2}}{|x-y|^{n}}\right\} \leq G_{D}(x, y) \\
& \leq C \min \left\{\frac{1}{|x-y|^{n-\alpha}}, \frac{\delta(x)^{\alpha / 2} \delta(y)^{\alpha / 2}}{|x-y|^{n}}\right\} .
\end{aligned}
$$

In the Brownian motion case, the Poisson kernel in a bounded $C^{1,1}$ domain is the normal derivative of the Green function. In the case of an $\alpha$-symmetric process, $0<\alpha<2$, this kind of relationship can not be expected to hold. For $0<\alpha<2$, the symmetric $\alpha$-stable process has discontinuous sample paths and therefore the exit distribution of $X_{\tau_{D}}$ under $P_{x}$ does not concentrate on the boundary $\partial D$. In fact, we have the following

Theorem 1.4. For every bounded domain $D$ in $\mathbf{R}^{n}$ satisfying uniform exterior cone condition, there is a function $K_{D}(x, z)$ defined on $D \times D^{c}$ such that

$$
E_{x}\left[\varphi\left(X_{\tau_{D}}\right)\right]=A(n, \alpha) \int_{D^{c}} K_{D}(x, z) \varphi(z) d z, \quad x \in D
$$

for every $\varphi \geq 0$ on $D^{c}$, where

$$
A(n, \alpha)=\frac{\alpha 2^{\alpha-1} \Gamma\left(\frac{\alpha+n}{2}\right)}{\pi^{n / 2} \Gamma\left(1-\frac{\alpha}{2}\right)} .
$$

Furthermore

$$
K_{D}(x, z)=A(n, \alpha) \int_{D} \frac{G_{D}(x, y)}{|y-z|^{n+\alpha}} d y, \quad x \in D, y \in D^{c} .
$$

Recall that a domain $D$ in $\mathbf{R}^{n}$ is said to satisfy the uniform exterior cone condition if there exist constants $\eta>0, r>0$ and a cone $\mathscr{C}=\left\{x=\left(x_{1}, \ldots, x_{n}\right) \in\right.$ $\left.\mathbf{R}^{n}: 0<x_{n},\left(x_{1}^{2}+\cdots+x_{n-1}^{2}\right)^{1 / 2}<\eta x_{n}\right\}$ such that for every $z \in \partial D$, there is a cone $\mathscr{C}_{z}$ with vertex $z$, isometric to $\mathscr{C}$ and satisfying $\mathscr{C}_{z} \cap B(z, r) \subset D^{c}$. It is well known that bounded $C^{1,1}$ domains satisfy the uniform exterior cone condition, therefore the above theorem holds in particular for bounded $C^{1,1}$ domains. In principle, by using Theorem 1.4 and the bounds for the Green functions, one could get two-sided bounds on the Poisson kernels. However, it turns out to be a pretty challenging task.

Theorem 1.5. Suppose that $D$ is a bounded $C^{1,1}$ domain in $\mathbf{R}^{n}$. Then there exists $a C=C(D, \alpha)>1$ such that for $x \in D$ and $z \in \bar{D}^{c}$,

$$
\begin{aligned}
\frac{\delta(x)^{\alpha / 2}}{C \delta(z)^{\alpha / 2}(1+\delta(z))^{\alpha / 2}} \frac{1}{|x-z|^{n}} & \leq K_{D}(x, z) \\
& \leq \frac{C \delta(x)^{\alpha / 2}}{\delta(z)^{\alpha / 2}(1+\delta(z))^{\alpha / 2}} \frac{1}{|x-z|^{n}} .
\end{aligned}
$$


For the corresponding results for Brownian motion, see Zhao [22]. Using the bounds above, we have

Theorem 1.6 ((3G Theorem)). Suppose that $D$ is a bounded $C^{1,1}$ domain in $\mathbf{R}^{n}$. Then there exists a $C=C(D, \alpha)>0$ such that

$$
\begin{aligned}
\frac{G_{D}(x, y) G_{D}(y, w)}{G_{D}(x, w)} \leq & C \frac{|x-w|^{n-\alpha}}{|x-y|^{n-\alpha}|y-w|^{n-\alpha}}, \\
& x, y, w \in D . \\
\frac{G_{D}(x, y) K_{D}(y, z)}{K_{D}(x, z)} \leq & C \frac{|x-z|^{n-\alpha}}{|x-y|^{n-\alpha}|y-z|^{n-\alpha}}, \\
& x, y \in D, z \in \bar{D}^{c} .
\end{aligned}
$$

The estimates above are very useful and have a lot of applications. As an example of these applications, we are going to prove that the conditional lifetimes for $X$ in a bounded $C^{1,1}$ domain are uniformly bounded. As another application of our estimates we are also going to give a simple proof of the boundary Harnack principle for nonnegative functions which are harmonic in a bounded $C^{1,1}$ domain $D$. Recently, the boundary Harnack principle for nonnegative functions which are harmonic in a bounded Lipschitz domain $D$ was proved by Bogdan [4]. To state these results, we first we need some definitions.

Definition 1.1. Let $D$ be a domain in $\mathbf{R}^{n}$. A locally integrable function $f$ defined on $\mathbf{R}^{n}$ satisfying the condition $\int_{\{|x|>1\}}|f(x)||x|^{-(n+\alpha)} d x<\infty$ is said to be

1) $(-\Delta)^{\alpha / 2}$-superharmonic in $D$ if $f$ is lower semicontinuous in $D$ and for each $x \in D$ and each ball $B(x, r)$ with $\overline{B(x, r)} \subset D$,

$$
f(x) \geq E_{x} f\left(X\left(\tau_{B(x, r)}\right)\right) .
$$

2) $(-\Delta)^{\alpha / 2}$-harmonic in $D$ if $f$ is continuous in $D$ and for each $x \in D$ and each ball $B(x, r)$ with $\overline{B(x, r)} \subset D$,

$$
f(x)=E_{x} f\left(X\left(\tau_{B(x, r)}\right)\right) .
$$

Theorem 1.7 (Boundary Harnack Principle). Suppose that $D$ is a bounded $C^{1,1}$ domain in $\mathbf{R}^{n}, V$ is an open set of $\mathbf{R}^{n}$ and $K$ is a compact subset of $V$. Then there is a constant $C=C(D, V, K, \alpha)>0$ such that for any two $(-\Delta)^{\alpha / 2}-$ harmonic functions $u, v$ in $D$ which are strictly positive and bounded on $V \cap D$, and vanish on $V \cap D^{c}$, we have

$$
\frac{u(x)}{v(x)} \leq C \frac{u(y)}{v(y)}, \quad x, y \in K \cap D .
$$


For fixed $y \in D$ and $z \in \bar{D}^{c}$, it is easy to check that $\left\{G_{D}\left(X_{t}^{D}, y\right), \mathscr{F}_{t}^{D}\right\}_{t \geq 0}$ and $\left\{K_{D}\left(X_{t}^{D}, z\right), \mathscr{F}_{t}^{D}\right\}_{t>0}$ are nonnegative $P_{x}$-supermartingales for each $x \in \bar{D}$. So if we extend $G_{D}(\cdot, y)$ and $K_{D}(\cdot, z)$ to be zero outside $D$, then $G_{D}(\cdot, y)$ and $K_{D}(\cdot, z)$ are $(-\Delta)^{\alpha / 2}$-superharmonic in $D$.

We can take the $h$-transform of $X^{D}$, where $h(x)$ is taken to be $G_{D}(x, y)$ or $K_{D}(x, z)$. For each $y \in D$, the $G(\cdot, y)$-transformed process of $X^{D}$ is called the $y$-conditioned symmetric stable process whose state space is $(D \backslash\{y\}) \cup\{\partial\}$, where $\partial$ is the added cemetery point. The lifetime of the conditional process is $\zeta=\tau_{D \backslash\{y\}}$. We continue to use $X_{t}^{D}$ to denote the generic random variable of the conditional process, but use $P_{x}^{y}$ and $E_{x}^{y}$ to denote its probability and expectation respectively. For $z \in \bar{D}^{c}$, the $K(\cdot, z)$-transformed process is called the $z$-conditioned symmetric stable process whose state space is $D \cup\{\partial\}$ and its lifetime is $\zeta=\tau_{D}$. Its probability and expectation are denoted as $P_{x}^{z}$ and $E_{x}^{z}$ respectively. Using Theorem 1.6, one can show easily that any bounded $C^{1,1}$ domain is a Cranston-McConnell domain for symmetric stable process. That is,

Theorem 1.8 (Conditional Lifetimes). Suppose that $D$ is a bounded $C^{1,1}$ domain in $\mathbf{R}^{n}$. Then

$$
\sup _{z \in \bar{D}^{c}, x \in D} E_{x}^{z}\left(\tau_{D}\right)<\infty \quad \text { and } \sup _{y \in D, x \in D \backslash\{y\}} E_{x}^{y}\left(\tau_{D \backslash\{y\}}\right)<\infty .
$$

In [5], we apply the estimates in Theorems 1.1-1.2 and Theorem 1.5 to show that logarithmic Sobolev inequality and intrinsic ultracontractivity hold for symmetric $\alpha$-stable processes in bounded $C^{1,1}$ domains. We then use these to establish the conditional gauge theorem for symmetric $\alpha$-stable processes.

The rest of the paper is organized as follows. In Sect. 2 we prove the upper bound estimates Theorem 1.1 for Green function $G_{D}$. Due to the length of its argument, the proof for Theorem 1.2 is postponed until Sect. 6. Theorem 1.4 and 1.5 are proved in Sect. 3. The boundary Harnack principle is proved in Sect. 4, the $3 G$ Theorem and conditional lifetime Theorem are proved in Sect. 5.

In the sequel we use $\omega_{n}$ to denote the surface area of the unit ball in $\mathbf{R}^{n}$. That is, $\omega_{n}=2 \pi^{n / 2} \Gamma\left(\frac{n}{2}\right)^{-1}$. In the proofs of this paper, constants $c$ and $C$, which do not change their dependence, may change their values from line to line. The Lebesgue measure of a Borel measurable set $A$ will be denoted as $|A|$. For a bounded domain $D$ in $\mathbf{R}^{n}$, we use $d_{D}$ to denote the diameter of $D$.

Although the main results of this paper are stated for bounded $C^{1,1}$ domains only, the assumption about the connectedness of $D$ is not really needed in the proof. All our proofs will go through if $D$ has a finite number of components $D_{i}$ such that each $D_{i}$ is bounded $C^{1,1}$ and that $\overline{D_{i}}$ and $\bar{D}_{j}$ are disjoint for $i \neq j$. 


\section{Upper bound estimates for Green functions}

It is easy to see from the strong Markov property of $X$ that for each $y \in D$ and $z \in \bar{D}^{c}, G_{D}(\cdot, y)$ and $K_{D}(\cdot, z)$ are superharmonic functions in $D$. If $f$ and $g$ are superharmonic, then so is $f \wedge g$. If $f$ is superharmonic in $D$ and $f \geq 0$ on $D^{c}$, then $f \geq 0$ on $\mathbf{R}^{n}$ by the limit theorem for supermartingales.

The following proposition follows immediately from the scaling property of the symmetric stable processes, so we will omit the proof.

Proposition 2.1. Let $D$ be a domain in $\mathbf{R}^{n}$ and $a>0$ be a constant. Then

$$
G_{D}(x, y)=a^{\alpha-n} G_{D / a}(x / a, y / a), \quad x, y \in D .
$$

Lemma 2.2. There exists a constant $C=C(n, \alpha)>0$ such that for any ball $B \subset \mathbf{R}^{n}$ we have

$$
G_{B}(x, y) \leq C \quad \frac{\delta_{B}(x)^{\alpha / 2} \delta_{B}(y)^{\alpha / 2}}{|x-y|^{n}}, x, y \in B,
$$

where $G_{B}$ is the Green function of $B$ and $\delta_{B}(x)=d(x, \partial B)$.

Proof. We first consider the case that $B=B(0,1)$. It follows from [3] that

$$
G_{B}(x, y)=2^{-\alpha} \pi^{-n / 2} \Gamma\left(\frac{\alpha}{2}\right)^{-2} \Gamma\left(\frac{n}{2}\right) \int_{0}^{z}(u+1)^{-n / 2} u^{\alpha / 2-1} d u|x-y|^{\alpha-n}
$$

where $z=\left(1-|x|^{2}\right)\left(1-|y|^{2}\right)|x-y|^{-2}$. Note that

$$
\begin{aligned}
& \int_{0}^{z}(u+1)^{-n / 2} u^{\alpha / 2-1} d u \\
= & z^{\alpha / 2} \int_{0}^{1}(1+v z)^{-n / 2} v^{\alpha / 2-1} d v \\
= & (1-|x|)^{\alpha / 2}(1-|y|)^{\alpha / 2}(1+|x|)^{\alpha / 2}(1+|y|)^{\alpha / 2}|x-y|^{-\alpha} . \\
& \cdot \int_{0}^{1}(1+v z)^{-n / 2} v^{\alpha / 2-1} d v \\
\leq & \left(\int_{0}^{1}(1+v z)^{-n / 2} v^{\alpha / 2-1} d v\right) 2^{\alpha} \delta_{B}(x)^{\alpha / 2} \delta_{B}(y)^{\alpha / 2}|x-y|^{-\alpha} .
\end{aligned}
$$

Since

$$
\int_{0}^{1}(1+v z)^{-n / 2} v^{\alpha / 2-1} d v<\int_{0}^{1} v^{\alpha / 2-1} d v<\infty
$$

the assertion of Lemma 2.2 holds for $B=B(0,1)$. For $B=B(0, r)$ with radius $r$, by Proposition 2.1, $G_{B(0, r)}(x, y)=r^{-n+\alpha} G_{B(0,1)}\left(\frac{x}{r}, \frac{y}{r}\right)$, we have

$$
G_{B(0, r)} \leq C \frac{\delta_{B}(x)^{\alpha / 2} \delta_{B}(y)^{\alpha / 2}}{|x-y|^{n}}, \quad \forall x, y \in B .
$$


Lemma 2.3. There exists $C=C(n, \alpha)>0$ such that for any ball $B \subset \mathbf{R}^{n}$ we have

$$
G_{B}(x, y) \leq C \frac{\delta_{B}(x)^{\alpha / 2}}{\delta_{B}(y)^{\alpha / 2}|x-y|^{n-\alpha}}, \quad x, y \in B .
$$

Proof. Let $G(x, y)$ be the Green function of the whole space. Clearly

$$
G_{B}(x, y) \leq G(x, y) .
$$

Combining this with Lemma 2.2 and formula (1.1), we have

$$
\begin{aligned}
G_{B}(x, y) & \leq G(x, y) \wedge\left(C \frac{\delta_{B}(x)^{\alpha / 2} \delta_{B}(y)^{\alpha / 2}}{|x-y|^{n}}\right) \\
& \leq \frac{C \delta_{B}(x)^{\alpha / 2}}{\delta_{B}(y)^{\alpha / 2}|x-y|^{n-\alpha}}\left(\frac{\delta_{B}(y)^{\alpha / 2}}{\delta_{B}(x)^{\alpha / 2}} \wedge \frac{\delta_{B}(y)^{\alpha}}{|x-y|^{\alpha}}\right) .
\end{aligned}
$$

If $\delta_{B}(y)>2 \delta_{B}(x)$, then $|x-y| \geq \delta_{B}(y)-\delta_{B}(x)>\frac{1}{2} \delta_{B}(y)$ and so

$$
\frac{\delta_{B}(y)^{\alpha / 2}}{\delta_{B}(x)^{\alpha / 2}} \wedge \frac{\delta_{B}(y)^{\alpha}}{|x-y|^{\alpha}} \leq \frac{\delta_{B}(y)^{\alpha}}{|x-y|^{\alpha}} \leq 2^{\alpha}
$$

If $\delta_{B}(y) \leq 2 \delta_{B}(x)$, then

$$
\frac{\delta_{B}(y)^{\alpha / 2}}{\delta_{B}(x)^{\alpha / 2}} \wedge \frac{\delta_{B}(y)^{\alpha}}{|x-y|^{\alpha}} \leq \frac{\delta_{B}(y)^{\alpha / 2}}{\delta_{B}(x)^{\alpha / 2}} \leq 2^{\alpha / 2}
$$

Lemma 2.3 is thus proved.

Lemma 2.4. There exists a constant $C=C(n, \alpha)>0$ such that for any ball $B \subset \mathbf{R}^{n}$ we have

$$
G_{B}(x, y) \leq C \frac{\delta_{B}(x)^{\alpha / 2}}{|x-y|^{n-\alpha / 2}}, \quad x, y \in B
$$

Proof. Clearly Lemma 2.4 holds for $x=y$. For $x, y \in B$ with $x \neq y$, by Lemma 2.2 and Lemma 2.3,

$$
G_{B}(x, y) \leq \frac{C \delta_{B}(x)^{\alpha / 2}}{|x-y|^{n-\alpha / 2}}\left(\frac{|x-y|^{\alpha / 2}}{\delta_{B}(y)^{\alpha / 2}} \wedge \frac{\delta_{B}(y)^{\alpha / 2}}{|x-y|^{\alpha / 2}}\right) \leq \frac{C \delta_{B}(x)^{\alpha / 2}}{|x-y|^{n-\alpha / 2}} .
$$

Lemma 2.5. There exists a constant $C=C(n, \alpha)>0$ such that for any $B=B(a, r) \subset \mathbf{R}^{n}$ we have

$$
G_{B^{c}}(x, y) \leq C|y-a|^{\alpha / 2} \frac{\delta_{B}(x)^{\alpha / 2}}{|x-y|^{n-\alpha / 2}}, \quad x, y \in B^{c},
$$

where $G_{B^{c}}$ is the Green function of $B^{c}$. 
Proof. In view of Proposition 2.1, we may assume that $B=B(0,1)$. Then from the discussions on pages 263-265 of [16] one can easily show that

$$
G_{B^{c}}(x, y)=\left|x^{*}\right|^{n-\alpha}\left|y^{*}\right|^{n-\alpha} G_{B}\left(x^{*}, y^{*}\right)
$$

where $x^{*}=x /|x|^{2}$ and $y^{*}=y /|y|^{2}$. Since

$$
\left|x^{*}-y^{*}\right|=\frac{|x-y|}{|x||y|} \quad \text { and } \quad \delta_{B}\left(x^{*}\right)=\frac{\delta_{B}(x)}{|x|},
$$

we have by Lemma 2.4 that

$$
\begin{aligned}
G_{B^{c}}(x, y) & \leq C\left|x^{*}\right|^{n-\alpha}\left|y^{*}\right|^{n-\alpha} \frac{\delta_{B}\left(x^{*}\right)^{\alpha / 2}}{\left|x^{*}-y^{*}\right|^{n-\alpha / 2}} \\
& =C|y|^{\alpha / 2} \frac{\delta_{B}(x)^{\alpha / 2}}{|x-y|^{n-\alpha / 2}} .
\end{aligned}
$$

Now let $D$ be a bounded $C^{1,1}$ domain and let $G_{D}$ be the Green function of $D$. It is well known that there exists $r_{0}>0$ depending only on $D$ such that for any $z \in \partial D, 0<r \leq r_{0}$, there exist two balls $B_{1}^{z}(r)$ and $B_{2}^{z}(r)$ of radius $r$ such that $B_{1}^{z}(r) \subset D, B_{2}^{z}(r) \subset \mathbf{R}^{n} \backslash \bar{D}$ and $\{z\}=\partial B_{1}^{z}(r) \cap \partial B_{2}^{z}(r)$. Let $d_{D}$ be the diameter of $D$. In the following, we are going to assume that $r_{0}<\frac{1}{2} d_{D}$.

Proof of (1.4). Let $x_{0} \in \partial D$ be such that $\left|x-x_{0}\right|=\delta(x)$. Consider the ball $B=B_{2}^{x_{0}}\left(r_{0}\right)=B\left(a, r_{0}\right)$. From Lemma 2.5, there is a constant $C=C(n, \alpha)>0$ such that

$$
\begin{aligned}
G_{D}(x, y) & \leq G_{B^{c}}(x, y) \leq C|y-a|^{\alpha / 2} \frac{\delta_{B}(x)^{\alpha / 2}}{|x-y|^{n-\alpha / 2}} \\
& =C|y-a|^{\alpha / 2} \frac{\delta(x)^{\alpha / 2}}{|x-y|^{n-\alpha / 2}} .
\end{aligned}
$$

Proof of (1.6). From (1.3) and (1.4) we know that

$$
\begin{aligned}
G_{D}(x, y) & \leq C_{1} \frac{1}{|x-y|^{n-\alpha}}, \\
G_{D}(x, y) & \leq C_{2} \frac{\delta(x)^{\alpha / 2}}{|x-y|^{n-\alpha / 2}}
\end{aligned}
$$

Since $\delta(y) \leq \delta(x)+|x-y|$, we have $\delta(y)^{\alpha / 2} \leq \delta^{\alpha / 2}(x)+|x-y|^{\alpha / 2}$. Therefore,

$$
\begin{aligned}
\delta(y)^{\alpha / 2} G_{D}(x, y) & \leq \delta(x)^{\alpha / 2} G_{D}(x, y)+|x-y|^{\alpha / 2} G_{D}(x, y) \\
& \leq C_{1} \frac{\delta(x)^{\alpha / 2}}{|x-y|^{n-\alpha}}+C_{2} \frac{\delta(x)^{\alpha / 2}}{|x-y|^{n-\alpha}} .
\end{aligned}
$$


Hence

$$
G_{D}(x, y) \leq\left(C_{1}+C_{2}\right) \frac{\delta(x)^{\alpha / 2}}{\delta(y)^{\alpha / 2}} \frac{1}{|x-y|^{n-\alpha}}
$$

Proof of (1.5). If $\delta(y) \geq r_{0}$ or $\delta(y)<r_{0}$ and $|x-y| \leq 8 \delta(y)$, then (1.5) follows from (1.4). So we assume that $\delta(y)<r_{0}$ and $|x-y|>8 \delta(y)$. Set $r=\min \left(|x-y| / 8, r_{0}\right)$. Let $y_{0} \in \partial D$ be such that $\left|y-y_{0}\right|=\delta(y)$ and let $B(a, r)=B_{1}^{y_{0}}(r)$. Without loss of generality, we can assume that $a$ is at the origin and that $y_{0}=(0, \cdots, 0,-r)$. Then $B_{2}^{y_{0}}(r)=B(b, r)$ where $b=(0, \cdots, 0,-2 r)$. By the explicit formula for the Poisson kernel on the ball $B(0, r)$ given in [3] and the strong Markov property, we have

$$
\begin{aligned}
G_{D}(x, y) & =E_{y}\left[G_{D}\left(x, X_{\tau_{B(0, r)}}^{D}\right)\right] \\
& =C \int_{D \backslash B(0, r)} \frac{\left(r^{2}-|y|^{2}\right)^{\alpha / 2}}{\left(|u|^{2}-r^{2}\right)^{\alpha / 2}} \frac{1}{|y-u|^{n}} G_{D}(x, u) d u \\
& \leq C \delta(x)^{\alpha / 2} \delta(y)^{\alpha / 2} \int_{D \backslash B(0, r)} \frac{d u}{(|u|-r)^{\alpha / 2}|y-u|^{n}|x-u|^{n-\frac{\alpha}{2}}} \\
& \leq C \delta(x)^{\alpha / 2} \delta(y)^{\alpha / 2} \int_{D_{0}} \frac{d u}{(|u|-r)^{\alpha / 2}|u-y|^{n}|u-x|^{n-\frac{\alpha}{2}}},
\end{aligned}
$$

where in the first inequality above we used (1.4) and in the last expression above $D_{0}$ is the set $B(b, r)^{c} \backslash B(0, r)$. Let $u=r v, \tilde{x}=\frac{x}{r}, \tilde{y}=\frac{y}{r}$. Then

$$
\int_{D_{0}} \frac{d u}{(|u|-r)^{\alpha / 2}|u-y|^{n}|u-x|^{n-\frac{\alpha}{2}}}=\frac{1}{r^{n}} \int_{D_{1}} \frac{d v}{(|v|-1)^{\alpha / 2}|v-\tilde{y}|^{n}|v-\tilde{x}|^{n-\frac{\alpha}{2}}}
$$

where $\left.D_{1}=\frac{1}{r} D_{0}=B((0, \cdots, 0,-2), 1)^{c} \backslash B(0,1)\right)$. Note that $|\tilde{y}|<1$ and $|\tilde{x}|>7$. The proof will be finished if we can show that the function

$$
F(x, y)=\int_{D_{1}} \frac{d v}{(|v|-1)^{\alpha / 2}|v-y|^{n}|v-x|^{n-\frac{\alpha}{2}}}
$$

is bounded on the set

$$
\overline{B((0, \cdots, 0,-2), 1)^{c} \cap\{|x|>7\}} \times \overline{B(0,1) \cap\left\{\left(0, \cdots, 0, y_{n}\right): y_{n}<0\right\}}
$$

by a constant depending only on $D$ and $\alpha$. In order to accomplish this, one only has to show that the function $F(x,(0, \cdots, 0,-1))$ is bounded on the set $\overline{B((0, \cdots, 0,-2), 1)^{c} \cap\{|x|>7\}}$, which can be accomplished by elementary analysis. We omit the details here. 


\section{Poisson kernel estimates}

Let $D$ be a bounded domain in $\mathbf{R}^{n}$ and let $X$ be the symmetric $\alpha$-stable process on $\mathbf{R}^{n}$. In this Sect. we are going to prove Theorem 1.4 first, and then we will use Theorem 1.4 to prove Theorem 1.5. To prove Theorem 1.4, we need the following result:

Theorem 3.1. For any Borel measurable $\phi \geq 0$ on $D^{c}$

$$
E_{x}\left[\phi\left(X_{\tau_{D}}\right) ; X_{\tau_{D}} \neq X_{\tau_{D}-}\right]=\int_{D^{c}} \phi(z) d z \int_{D} \frac{G_{D}(x, y)}{|y-z|^{n+\alpha}} d y .
$$

Proof. It is known (see, e.g., page 19 of [2]) that the Lévy system $(N, H)$ of the $\alpha$-symmetric stable process $X$ is given by

$$
N(x, d y)=A(n, \alpha) \frac{1}{|x-y|^{n+\alpha}} d y, \quad H_{t}=t
$$

where $A(n, \alpha)$ is given by (1.10). Extend $\phi$ to $\mathbf{R}^{n}$ by defining it to be zero in $D$. For any non-negative Borel measurable function $f$ on $\mathbf{R}^{n}$, set

$$
F(x)=A(n, \alpha) \int_{\mathbf{R}^{n}} \frac{f(x) \phi(y)}{|x-y|^{n+\alpha}} d y=A(n, \alpha) \int_{D^{c}} \frac{f(x) \phi(y)}{|x-y|^{n+\alpha}} d y .
$$

Define

$$
\kappa(\omega, d t)=\sum_{s>0} f\left(X_{s-}(\omega)\right) \phi\left(X_{s}(\omega)\right) \delta_{s}(d t) 1_{\left\{X_{s-}(\omega) \neq X_{s}(\omega)\right\}} .
$$

By Theorems (73.1) and (73.4) of [19] we know that the dual predictable projection of $\kappa$ is given by

$$
\kappa^{p}(d t)=F\left(X_{t}\right) d t .
$$

Since the random process $1_{\left\{(t, \omega): 0<t \leq \tau_{D}(\omega)\right\}}$ is left-continuous and is therefore predictable,

$$
\begin{aligned}
E_{x} \sum_{0<s \leq \tau_{D}} f\left(X_{s-}\right) \phi\left(X_{s}\right) 1_{\left\{X_{s-} \neq X_{s}\right\}} & =E_{x} \int_{0}^{\tau_{D}} F\left(X_{s}\right) d s \\
& =\int_{D} G_{D}(x, y) F(y) d y \\
& =A(n, \alpha) \int_{D} G_{D}(x, y) \int_{D^{c}} \frac{f(y) \phi(z)}{|y-z|^{n+\alpha}} d z d y \\
& =A(n, \alpha) \int_{D^{c}} \phi(z) d z \int_{D} \frac{G_{D}(x, y) f(y)}{|y-z|^{n+\alpha}} d y
\end{aligned}
$$

Therefore

$$
E_{x}\left(f\left(X_{\tau_{D}-}\right) \phi\left(X_{\tau_{D}}\right) 1_{\left\{X_{\tau_{D}-} \neq X_{\tau_{D}}\right\}}\right)=A(n, \alpha) \int_{D^{c}} \phi(z) d z \int_{D} \frac{G_{D}(x, y) f(y)}{|y-z|^{n+\alpha}} d y .
$$

In particular, 


$$
E_{x}\left[\phi\left(X_{\tau_{D}}\right) ; X_{\tau_{D}} \neq X_{\tau_{D}-}\right]=A(n, \alpha) \int_{D^{c}} \phi(z) d z \int_{D} \frac{G_{D}(x, y)}{|y-z|^{n+\alpha}} d y
$$

Proof of Theorem 1.4. It follows from Lemma 6 of Bogdan [4] that for any bounded domain $D$ satisfying uniform exterior cone condition,

$$
P_{x}\left(X_{\tau_{D}} \neq X_{\tau_{D}-}\right)=1, \quad x \in D .
$$

The theorem now follows immediately from (3.1) and (3.2).

Theorem 3.2. Suppose $D$ is a bounded $C^{1,1}$ domain in $\mathbf{R}^{n}$. Then there is a constant $C=C(D, \alpha)>0$ such that

$$
K_{D}(x, z) \leq C \frac{\delta(x)^{\alpha / 2}}{(1+\delta(z))^{\alpha / 2} \delta(z)^{\alpha / 2}} \frac{1}{|x-z|^{n}}, \quad x \in D, z \in \bar{D}^{c} .
$$

Proof. By Theorem 1.4

$$
\begin{aligned}
K_{D}(x, z) & =A(n, \alpha) \int_{D} \frac{G_{D}(x, y)}{|y-z|^{n+\alpha}} d y \\
& \leq A(n, \alpha) \frac{2^{n}}{|x-z|^{n}} \int_{D} \frac{G_{D}(x, y)}{|y-z|^{n+\alpha}}\left(|x-y|^{n}+|y-z|^{n}\right) d y \\
& \leq A(n, \alpha) \frac{2^{n}}{|x-z|^{n}}\left(\int_{D} \frac{G_{D}(x, y)|x-y|^{n}}{|y-z|^{n+\alpha}} d y+\int_{D} \frac{G_{D}(x, y)}{|y-z|^{\alpha}} d y\right) \\
& =A(n, \alpha) \frac{2^{n}}{|x-z|^{n}}(I+I I) .
\end{aligned}
$$

By Theorem 1.1, there is a constant $C=C(D, \alpha)>0$ such that

$$
G_{D}(x, y) \leq C \frac{\delta(x)^{\alpha / 2} \delta(y)^{\alpha / 2}}{|x-y|^{n}}
$$

We have

$$
\begin{aligned}
I & \leq C \delta(x)^{\alpha / 2} \int_{D} \frac{\delta(y)^{\alpha / 2}}{|y-z|^{n+\alpha}} d y \\
& \leq C \delta(x)^{\alpha / 2} \int_{D} \frac{1}{|y-z|^{n+\alpha / 2}} d y \\
& \leq C \delta(x)^{\alpha / 2} \omega_{n} \int_{\delta(z)}^{\delta(z)+d_{D}} \frac{1}{r^{n+\alpha / 2}} r^{n-1} d r \\
& \leq\left.\frac{2 C \omega_{n}}{\alpha} \delta(x)^{\alpha / 2}\left(-r^{-\alpha / 2}\right)\right|_{\delta(z)} ^{\delta(z)+d_{D}} \\
& \leq \frac{2 C \omega_{n}}{\alpha} \frac{\delta(x)^{\alpha / 2}}{\delta(z)^{\alpha / 2}} .
\end{aligned}
$$

Using 


$$
G_{D}(x, y) \leq C \frac{\delta(x)^{\alpha / 2}}{|x-y|^{n-\alpha / 2}}
$$

we get

$$
\begin{aligned}
I I \leq & C \delta(x)^{\alpha / 2} \int_{D} \frac{1}{|x-y|^{n-\alpha / 2}|y-z|^{\alpha}} d y \\
\leq & C \delta(x)^{\alpha / 2}\left\{\int_{\{y \in D:|x-y| \geq|y-z|\}} \frac{1}{|y-z|^{n+\alpha / 2}} d y+\right. \\
& \left.+\int_{\{y \in D:|x-y|<|y-z|\}} \frac{1}{|x-y|^{n-\alpha / 2}} \frac{1}{|y-z|^{\alpha}} d y\right\} \\
\leq & C \delta(x)^{\alpha / 2}\left\{\frac{1}{\delta(z)^{\alpha / 2}}\right. \\
& \left.+\int_{\{y \in D:|x-y|<|y-z|\}} \frac{1}{|x-y|^{n-\alpha / 2}(|| x-z|-| x-y|| \vee|x-y|)^{\alpha}} d y\right\} .
\end{aligned}
$$

In the last inequality, we used (3.4) and the triangle inequality. Using spherical coordinates centered at $x$,

$$
\begin{aligned}
I I & \leq C \delta(x)^{\alpha / 2}\left\{\frac{1}{\delta(z)^{\alpha / 2}}+\int_{0}^{d_{D}} \frac{r^{n-1}}{r^{n-\alpha / 2}(|a-r| \vee r)^{\alpha}} d r\right\} \\
& \stackrel{r=a s}{\leq} c \delta(x)^{\alpha / 2}\left\{\frac{1}{\delta(z)^{\alpha / 2}}+\frac{1}{a^{\alpha / 2}} \int_{0}^{d_{D} / a} \frac{s^{\alpha / 2-1}}{(|1-s| \vee s)^{\alpha}} d s\right\} .
\end{aligned}
$$

Since $0<\alpha<2$,

$$
\int_{0}^{d_{D} / a} \frac{s^{\alpha / 2-1}}{(|1-s| \vee s)^{\alpha}} d s \leq \int_{0}^{\infty} \frac{s^{\alpha / 2-1}}{(|1-s| \vee s)^{\alpha}} d s<\infty,
$$

thus

$$
I I \leq C \frac{\delta(x)^{\alpha / 2}}{\delta(z)^{\alpha / 2}}
$$

for some constant $C>0$. This together with (3.3) and (3.4), implies

$$
K_{D}(x, z) \leq A(n, \alpha) C \frac{\delta(x)^{\alpha / 2}}{\delta(z)^{\alpha / 2}} \frac{1}{|x-z|^{n}} \quad \text { for } \quad x \in D, z \in D^{c}
$$

In particular, if $z \in D^{c}$ with $\delta(z) \leq d_{D}$, then

$$
K_{D}(x, z) \leq A(n, \alpha) C\left(1+d_{D}\right)^{\alpha / 2} \frac{\delta(x)^{\alpha / 2}}{\delta(z)^{\alpha / 2}(1+\delta(z))^{\alpha / 2}} \frac{1}{|x-z|^{n}} .
$$

If $\delta(z)>d_{D}$, then for $y \in D, \delta(z) \leq|y-z| \leq 2 \delta(z)$. Therefore 


$$
\begin{aligned}
K(x, z) & =A(n, \alpha) \int_{D} \frac{G_{D}(x, y)}{|y-z|^{n+\alpha}} d y \\
& \leq A(n, \alpha) \frac{1}{\delta(z)^{n+\alpha}} \int_{D} G_{D}(x, y) d y \\
& \leq A(n, \alpha) \frac{1}{\delta(z)^{n+\alpha}} \int_{D} \frac{C_{1} \delta(x)^{\alpha / 2}}{|x-y|^{n-\alpha / 2}} d y \\
& \leq A(n, \alpha) C \frac{\delta(x)^{\alpha / 2}}{\delta(z)^{\alpha}} \frac{1}{\delta(z)^{n}} \\
& \leq A(n, \alpha) C \frac{\delta(x)^{\alpha / 2}}{\delta(z)^{\alpha}} \frac{1}{|x-z|^{n}} \\
& \leq A(n, \alpha) C \frac{\delta(x)^{\alpha / 2}}{\delta(z)^{\alpha / 2}(1+\delta(z))^{\alpha / 2}} \frac{1}{|x-z|^{n}} .
\end{aligned}
$$

This completes the proof of the theorem.

Before going to estimate the lower bound of $K_{D}$, we first record three simple facts about bounded Lipschitz domains in the following lemma. The proof of this lemma is straightforward and thus omitted. We say a Lipschitz domain $D$ has Lipschitz characteristic constants $\left(r_{0}, A_{0}\right)$ if for every $z \in \partial D$, there is a local coordinate system $\left(\xi_{1}, \xi^{(1)}\right) \in \mathbf{R} \times \mathbf{R}^{n-1}$ with origin sitting at $z$ and there is a Lipschitz function $f$ defined on $\mathbf{R}^{n-1}$ with Lipschitz constant $A_{0}$ such that $D \cap B\left(z, r_{0}\right)=B\left(z, r_{0}\right) \cap\left\{\xi=\left(\xi_{1}, \xi^{(1)}\right): \xi_{1}>f\left(\xi^{(1)}\right)\right\}$.

Lemma 3.3. (1) If $D$ is a Lipschitz domain with characteristic constants $\left(r_{0}, A_{0}\right)$ and $a>0$ is a constant, then the dilation $a D$ has Lipschitz characteristic constant $\left(a r_{0}, A_{0}\right)$.

(2) Suppose that $0 \in \bar{D}$ and $r>0,0<\alpha<2$. Then there is a constant $C_{0}>0$ depending only on $r, \alpha$, and the Lipschitz characteristic constants $\left(r_{0}, A_{0}\right)$ of $D$ such that

$$
\int_{D \cap B(0, r)} \frac{1}{|y|^{n-\alpha}}\left(1 \wedge \frac{\delta(y)^{\alpha / 2}}{|y|^{\alpha}}\right) d y \geq C_{0}>0 .
$$

(3) There is a constant $C_{0}=C_{0}\left(r_{0}, A_{0}, r\right)>0$ such that

$$
\int_{D \cap B(x, r)} \delta(y)^{\alpha / 2} d y \geq C_{0}>0 \text { for all } x \in \partial D .
$$

Theorem 3.4. Suppose $D$ is a bounded $C^{1,1}$ domain in $\mathbf{R}^{n}$. Then there is a constant $C=C(D, \alpha)>0$ such that

$$
K_{D}(x, z) \geq C \frac{\delta(x)^{\alpha / 2}}{\delta(z)^{\alpha / 2}(1+\delta(z))^{\alpha / 2}} \frac{1}{|x-z|^{n+\alpha}}, \quad x \in D, z \in \bar{D}^{c} .
$$

Proof. Let $\left(r_{0}, A_{0}\right)$ denote the Lipschitz characteristic constants for $D$. By Theorem 1.2, we have 


$$
G_{D}(x, y) \geq C \min \left\{\frac{1}{|x-y|^{n-\alpha}}, \frac{\delta(x)^{\alpha / 2} \delta(y)^{\alpha / 2}}{|x-y|^{n}}\right\}, \quad x, y \in D .
$$

Recall that $d_{D}$ is the diameter of $D$. We derive the estimate by considering two cases.

Case 1. $\delta(z) \leq d_{D}$.

Using change of variable, we have

$$
\begin{aligned}
& K_{D}(x, z) \\
& =A(n, \alpha) \int_{D} \frac{G_{D}(x, y)}{|y-z|^{n+\alpha}} d y \\
& \geq A(n, \alpha) C \delta(x)^{\alpha / 2} \int_{D} \frac{1}{|y-z|^{n+\alpha}} \frac{1}{|x-y|^{n-\alpha}}\left(\frac{1}{\delta(x)^{\alpha / 2}} \wedge \frac{\delta(y)^{\alpha / 2}}{|x-y|^{\alpha}}\right) d y \\
& \quad \sum^{y-x=|x-z| \widetilde{y}} A(n, \alpha) \frac{C \delta(x)^{\alpha / 2}}{|x-z|^{n+\alpha / 2}} \int_{D} \frac{1}{(1+|\widetilde{y}|)^{n+\alpha}} \frac{1}{|\widetilde{y}|^{n-\alpha}}\left(\frac{1}{\widetilde{\delta}(\widetilde{x})^{\alpha / 2}} \wedge \frac{\widetilde{\delta}(\widetilde{y})^{\alpha / 2}}{|\widetilde{y}|^{\alpha}}\right) d \widetilde{y}
\end{aligned}
$$

where $\widetilde{D}=\frac{1}{|x-z|}(D-x)$ and $\widetilde{\delta}(\widetilde{y})=d(\widetilde{y}, \partial \widetilde{D})$. Since $\widetilde{\delta}(\widetilde{x})=\frac{\delta(x)}{|x-z|} \leq 1$,

$$
K_{D}(x, z) \geq A(n, \alpha) \frac{C \delta(x)^{\alpha / 2}}{|x-z|^{n+\alpha / 2}} \int_{\widetilde{D}} \frac{1}{(1+|\widetilde{y}|)^{n+\alpha}} \frac{1}{|\widetilde{y}|^{n-\alpha}}\left(1 \wedge \frac{\widetilde{\delta}(\widetilde{y})^{\alpha / 2}}{|\widetilde{y}|^{\alpha}}\right) d \widetilde{y}
$$

Note that $|x-z| \leq 2 d_{D}$, so $\widetilde{D}$ has Lipschitz characteristic constants $\left(\frac{r_{0}}{2 d_{D}}, A_{0}\right)$. Let $0<r<\frac{r_{0}}{2 d_{D}}$ be fixed. Then

$$
\begin{aligned}
& K_{D}(x, z) \\
& \geq A(n, \alpha) \frac{C \delta(x)^{\alpha / 2}}{|x-z|^{n+\alpha / 2}} \int_{\widetilde{D} \cap B(0, r)} \frac{1}{(1+r)^{n+\alpha}} \frac{1}{|\widetilde{y}|^{n-\alpha}}\left(1 \wedge \frac{\widetilde{\delta}(\widetilde{y})^{\alpha / 2}}{|\widetilde{y}|^{\alpha}}\right) d \widetilde{y} \\
& \geq \frac{C \delta(x)^{\alpha / 2}}{|x-z|^{n+\alpha / 2}} \text { by Lemma 3.3(2). }
\end{aligned}
$$

$1^{\circ}$. If $\delta(z) \geq r_{0}$, then by $(3.5)$

$$
K_{D}(x, z) \geq A(n, \alpha) \frac{C r_{0}^{\alpha / 2}}{\delta(z)^{\alpha / 2}} \frac{\delta(x)^{\alpha / 2}}{|x-z|^{n}\left(2 d_{D}\right)^{\alpha / 2}} \geq A(n, \alpha) C \frac{\delta(x)^{\alpha / 2}}{\delta(z)^{\alpha / 2}|x-z|^{n}} .
$$

$2^{\circ}$. If $|x-z| \leq 4 \delta(z)$, then by (3.5)

$$
K_{D}(x, z) \geq A(n, \alpha) \frac{C \delta(x)^{\alpha / 2}}{|x-z|^{n}(4 \delta(z))^{\alpha / 2}} \geq A(n, \alpha) \frac{C \delta(x)^{\alpha / 2}}{\delta(z)^{\alpha / 2}|x-z|^{n}} .
$$

$3^{\circ}$. Lastly, if $|x-z|>4 \delta(z)$ and $\delta(z)<r_{0}$, then by a change of variable 


$$
\begin{aligned}
& K_{D}(x, z) \quad=\quad A(n, \alpha) \int_{D} \frac{G_{D}(x, y)}{|y-z|^{n+\alpha}} d y \\
& \geq \quad A(n, \alpha) C \delta(x)^{\alpha / 2} \int_{\left\{y \in D:|y-z|<\frac{1}{2}|x-z|\right\}} \frac{\delta(y)^{\alpha / 2}}{|x-y|^{n}|y-z|^{n+\alpha}} d y \\
& \stackrel{y-z=\delta(z) \widetilde{y}}{=} A(n, \alpha) \frac{C \delta(x)^{\alpha / 2}}{\delta(z)^{\alpha / 2}|x-z|^{n}} \int_{\left\{\widetilde{y} \in \widetilde{D}: \widetilde{y}\left|<\frac{1}{2}\right| \widetilde{x} \mid\right\}} \frac{|\widetilde{x}|^{n} \widetilde{\delta}(\tilde{y})^{\alpha / 2}}{|\widetilde{x}-\widetilde{y}|^{n}|\widetilde{y}|^{n+\alpha}} d \widetilde{y}
\end{aligned}
$$

where

$$
\widetilde{D}=\frac{1}{\delta(z)}(D-z) \text { and } \quad \widetilde{x}=\frac{x-z}{\delta(z)}
$$

Thus

$$
\begin{gathered}
K_{D}(x, z) \\
\quad \geq \quad A(n, \alpha) \frac{C \delta(x)^{\alpha / 2}}{\delta(z)^{\alpha / 2}|x-z|^{n}} \int_{\left\{\widetilde{y} \in \widetilde{D}: \widetilde{y}\left|<\frac{1}{2}\right| \widetilde{x} \mid\right\}} \frac{|\widetilde{x}|^{n} \widetilde{\delta}(\widetilde{y})^{\alpha / 2}}{(|\widetilde{x}|+|\widetilde{y}|)^{n}|\widetilde{y}|^{n+\alpha}} d \widetilde{y} \\
\stackrel{\text { since } \widetilde{x} \mid>4}{\rightarrow} \quad A(n, \alpha) \frac{C \delta(x)^{\alpha / 2}}{\delta(z)^{\alpha / 2}|x-z|^{n}} \int_{\{\widetilde{y} \in \widetilde{D}: \widetilde{y} \mid \leq 2\}} \widetilde{\delta}(\widetilde{y})^{\alpha / 2} d \widetilde{y} .
\end{gathered}
$$

Note that $\widetilde{D}$ has Lipschitz characteristic constants $\left(\frac{r_{0}}{\delta(z)}, A_{0}\right)$ with $\frac{r_{0}}{\delta(z)}>1$. Let $y^{*} \in \partial \widetilde{D}$ such that $\left|y^{*}\right|=d(0, \partial \widetilde{D})=1$. Then by (3.6), and Lemma 3.3(3),

$$
\begin{aligned}
K_{D}(x, z) & \geq A(n, \alpha) \frac{C \delta(x)^{\alpha / 2}}{\delta(z)^{\alpha / 2}|x-z|^{n}} \int_{\bar{D} \cap B\left(y^{*}, 1\right)} \widetilde{\delta}(\widetilde{y})^{\alpha / 2} d \widetilde{y} \\
& \geq A(n, \alpha) \frac{C \delta(x)^{\alpha / 2}}{\delta(z)^{\alpha / 2}|x-y|^{n}} .
\end{aligned}
$$

In summary, we have for $\delta(z) \leq d_{D}$,

$$
K_{D}(x, z) \geq A(n, \alpha) \frac{C \delta(x)^{\alpha / 2}}{\delta(z)^{\alpha / 2}|x-y|^{n}} \geq A(n, \alpha) \frac{C \delta(x)^{\alpha / 2}}{\delta(z)^{\alpha / 2}(1+\delta(z))^{\alpha / 2}} \frac{1}{|x-z|^{n}} .
$$

Case 2. $\delta(z)>d_{D}$

In this remaining case, for any $y \in D$, we have $\delta(z) \leq|y-z| \leq 2 \delta(z)$. Thus

$$
\begin{aligned}
K_{D}(x, z) & =A(n, \alpha) \int_{D} \frac{G_{D}(x, y)}{|y-z|^{n+\alpha}} d y \\
& \geq A(n, \alpha) \frac{1}{2^{n+\alpha} \delta(z)^{n+\alpha}} \int_{D} G_{D}(x, y) d y \\
& \geq A(n, \alpha) \frac{C \delta(x)^{\alpha / 2}}{\delta(z)^{n+\alpha}} \int_{D} \min \left\{\frac{1}{|x-y|^{n-\alpha} \delta(x)^{\alpha / 2}}, \frac{\delta(y)^{\alpha / 2}}{|x-y|^{n}}\right\} d y \\
& \geq A(n, \alpha) \frac{C \delta(x)^{\alpha / 2}}{\delta(z)^{\alpha}|x-z|^{n}} \int_{D}\left(\frac{1}{d_{D}^{\alpha / 2}|x-y|^{n-\alpha}} \wedge \frac{\delta(y)^{\alpha / 2}}{|x-y|^{n}}\right) d y .
\end{aligned}
$$


Note that

$$
\varphi(x)=\int_{D}\left(\frac{1}{d_{D}^{\alpha / 2}|x-y|^{n-\alpha}} \wedge \frac{\delta(y)^{\alpha / 2}}{|x-y|^{n}}\right) d y
$$

is strictly positive and continuous on $\bar{D}$, thus $\inf _{x \in D} \varphi(x) \geq C_{0}>0$. So by (3.7)

$$
K_{D}(x, z) \geq A(n, \alpha) \frac{C \delta(x)^{\alpha / 2}}{\delta(z)^{\alpha / 2}(1+\delta(z))^{\alpha / 2}} \frac{1}{|x-z|^{n}} .
$$

\section{Boundary Harnack inequality}

By using the strong Markov property and the quasi-left-continuity of $X$, one can show that if a function $u$ defined on $\mathbf{R}^{n}$ is $(-\Delta)^{\alpha / 2}$-harmonic in $D$, then for any open set $D_{1}$ with $\bar{D}_{1} \subset D$,

$$
u(x)=E_{x} u\left(X\left(\tau_{D_{1}}\right)\right), \quad \text { for } x \in D_{1} .
$$

Proof of Theorem 1.7. Without loss of generality, we can assume that for any $x \in D$,

$$
u(x)=E_{x} u\left(X\left(\tau_{D}\right)\right), v(x)=E_{x} v\left(X\left(\tau_{D}\right)\right) .
$$

Otherwise, we can take a $C^{1,1}$ domain $D_{1} \subset D$ and a set $V_{1}$ with $K \subset V_{1} \subset V$ such that $(\partial D) \cap V_{1} \subset(\partial D) \cap\left(\partial D_{1}\right) \subset V$. Then $u$ and $v$ are bounded and continuous in $D_{1}$ and vanishes in $D_{1}^{c} \cap V_{1}$. From Theorem 1.4 and the quasi-leftcontinuity of $X$ one can show that

$$
u(x)=E_{x} u\left(X\left(\tau_{D_{1}}\right)\right), v(x)=E_{x} v\left(X\left(\tau_{D_{1}}\right)\right) .
$$

Then we can work with $D_{1}, V_{1}, K$ instead of $K, V, D$.

Let $r_{0}=d\left(K \cap D, D^{c} \backslash V\right)>0$ and $d_{0}$ be the diameter of $D \cap K$. Then by Theorem 1.5 for $x \in D$

$$
\begin{aligned}
u(x) & =\int_{D^{c}} K_{D}(x, z) u(z) d z \\
& \geq C^{-1} \delta(x)^{\alpha / 2} \int_{D^{c}} \frac{u(z)}{\delta(z)^{\alpha / 2}(1+\delta(z))^{\alpha / 2}|x-z|^{n}} d z \\
& =C^{-1} \delta(x)^{\alpha / 2} \int_{D^{c} \backslash V} \frac{u(z)}{\delta(z)^{\alpha / 2}(1+\delta(z))^{\alpha / 2}|x-z|^{n}} d z
\end{aligned}
$$

Similarly

$$
u(x) \leq C \delta(x)^{\alpha / 2} \int_{D^{c} \backslash V} \frac{u(z)}{\delta(z)^{\alpha / 2}(1+\delta(z))^{\alpha / 2}|x-z|^{n}} d z .
$$

For $x, y \in D \cap K$ and $z \in D^{c} \backslash V$ 


$$
\begin{aligned}
|x-z| & \leq|y-z|+|x-y| \leq|y-z|+d_{0} \frac{r_{0}}{r_{0}} \\
& \leq|y-z|+\frac{d_{0}}{r_{0}}|y-z| \leq\left(1+\frac{d_{0}}{r_{0}}\right)|y-z| .
\end{aligned}
$$

Hence

$$
\frac{|x-z|}{|y-z|} \leq\left(1+\frac{d_{0}}{r_{0}}\right) \text {. }
$$

Interchange the role of $x$ and $y$, we have

$$
\left(1+\frac{d_{0}}{r_{0}}\right)^{-1} \leq \frac{|x-z|}{|y-z|} \leq 1+\frac{d_{0}}{r_{0}} .
$$

Thus by (4.1) and (4.2)

$$
C^{-1}\left(1+\frac{d_{0}}{r_{0}}\right)^{-n} \leq \frac{u(x)}{u(y)} \leq C\left(1+\frac{d_{0}}{r_{0}}\right)^{n} .
$$

Similarly

$$
C^{-1}\left(1+\frac{d_{0}}{r_{0}}\right)^{-n} \leq \frac{v(x)}{v(y)} \leq C\left(1+\frac{d_{0}}{r_{0}}\right)^{n} .
$$

Thus there is a constant $C=C(D, V, K, \alpha)>0$ such that

$$
\frac{u(x)}{v(x)} \leq C \frac{u(y)}{v(y)} \quad \text { for } x, y \in D \cap K .
$$

\section{3G Theorem and conditional lifetimes}

Proof of Theorem 1.6. For $x, y, w \in D$, if $|x-w|<\frac{1}{2} \max \{\delta(x), \delta(w)\}$, then by (1.3) and (1.8)

$$
\frac{G_{D}(x, y) G_{D}(y, w)}{G_{D}(x, w)} \leq C \frac{|x-w|^{n-\alpha}}{|x-y|^{n-\alpha}|y-w|^{n-\alpha}} .
$$

If $|x-w|>\frac{1}{2} \max \{\delta(x), \delta(w)\}$, then by (1.5), (1.7) and (1.9) we have

$$
\begin{aligned}
\frac{G_{D}(x, y) G_{D}(y, w)}{G_{D}(x, w)} & \leq C \frac{|x-w|^{n}}{\delta(x)^{\alpha / 2} \delta(w)^{\alpha / 2}} \frac{\delta(x)^{\alpha / 2} \delta(y)^{\alpha / 2}}{|x-y|^{n}} \frac{\delta(w)^{\alpha / 2}}{\delta(y)^{\alpha / 2}|y-w|^{n-\alpha}} \\
& =C \frac{|x-w|^{n}}{|x-y|^{n}|y-w|^{n-\alpha}}
\end{aligned}
$$

Interchange the role of $x$ and $w$,

$$
\frac{G_{D}(x, y) G_{D}(y, w)}{G_{D}(x, w)} \leq C \frac{|x-w|^{n}}{|x-y|^{n-\alpha}|y-w|^{n}} .
$$


If $|x-y| \geq \frac{1}{2}|x-w|$, we see from (5.1) that (1.11) holds. If $|x-y|<\frac{1}{2}|x-w|$, since $|y-w| \geq|x-w|-|x-y|>\frac{1}{2}|x-w|$, we get (1.1) from (5.2). Thus (1.11) is proved.

For $x, y \in D$ and $z \in \bar{D}^{c}$, by Theorem 1.5

$$
\frac{G_{D}(x, y) K_{D}(y, z)}{K_{0}(x, z)} \leq C G_{D}(x, y) \frac{\delta(y)^{\alpha / 2}}{\delta(x)^{\alpha / 2}} \frac{|x-z|^{n}}{|y-z|^{n}} .
$$

If $|x-z| \leq 2|y-z|$, by (5.3) and (1.6) we have

$$
\frac{G_{D}(x, y) K_{D}(y, z)}{K_{D}(x, z)} \leq C \frac{|x-z|^{n-\alpha}}{|x-y|^{n-\alpha}|y-z|^{n-\alpha}} .
$$

If $|x-z|>2|y-z|$, then $|x-z| \leq|x-y|+|y-z| \leq|x-y|+\frac{1}{2}|x-z|$. So $|x-z| \leq 2|x-y|$. By (4.3) and (1.5)

$$
\frac{G_{D}(x, y) K_{D}(x, y)}{K_{D}(x, y)} \leq C \frac{\delta(y)^{\alpha}}{|x-y|^{n}} \frac{|x-z|^{n}}{|y-z|^{n}} \leq C \frac{|x-z|^{n-\alpha}}{|x-y|^{n-\alpha}|y-z|^{n-\alpha}} .
$$

Proof of Theorem 1.8. For $y \in D$ and $x \in D \backslash\{y\}$, by (1.11)

$$
\begin{aligned}
E_{x}^{y}\left[\tau_{D \backslash\{y\}}\right] & =\int_{0}^{\infty} P_{x}^{y}\left(X_{t}^{D} \in D \backslash\{y\}\right) d t \\
& =\frac{1}{G_{D}(x, y)} \int_{0}^{\infty} E_{x}\left[G_{D}\left(X_{t}, y\right) ; X_{t}^{D} \in D \backslash\{y\}\right] d t \\
& =\int_{D} \frac{G_{D}(x, w) G_{D}(w, y)}{G_{D}(x, y)} d w \\
& \leq C \int_{D}\left(\frac{1}{|x-w|^{n-\alpha}}+\frac{1}{|y-w|^{n-\alpha}}\right) d w \\
& \leq 2 C \sup _{x \in \mathbf{R}^{n}} \int_{D} \frac{1}{|x-w|^{n-\alpha}} d w \\
& \equiv C_{0}<\infty .
\end{aligned}
$$

For $x \in D, z \in \bar{D}^{c}$, by (1.12)

$$
\begin{aligned}
E_{x}^{z}\left[\tau_{D}\right] & =\int_{D} \frac{G_{D}(x, w) K_{D}(w, z)}{K_{D}(x, z)} d w \\
& \leq C \int_{D}\left(\frac{1}{|x-w|^{n-\alpha}}+\frac{1}{|w-z|^{n-\alpha}}\right) d w \\
& \leq C_{0}<\infty .
\end{aligned}
$$




\section{Lower bound estimates for Green functions}

Since $D$ is a bounded $C^{1,1}$ domain, we know that there exist positive constants $C_{0}$ and $r_{0}$ depending only on $D$ such that for any $z, w \in \partial D,\left|n_{z}-n_{w}\right| \leq C_{0}|z-w|$ (where $n_{z}$ and $n_{w}$ are the inward unit normal vector to $\partial D$ at $z$ and $w$ respectively) and for any $z \in \partial D, 0<r \leq r_{0}$, there exist two balls $B_{1}^{z}(r)$ and $B_{2}^{z}(r)$ of radius $r$ such that $B_{1}^{z}(r) \subset D, B_{2}^{z}(r) \subset \mathbf{R}^{n} \backslash \bar{D}$ and $\partial B_{1}^{z}(r) \cap \partial B_{2}^{z}(r)=\{z\}$.

Without loss of generality, we can assume that

$$
r_{0} \leq \frac{1}{2 C_{0}}
$$

Lemma 6.1. For any $x \in[-1,1]$,

$$
(2 x-1)+\sqrt{(2 x-1)^{2}+4(1-x)^{2}} \geq 1 .
$$

Proof. Using first year calculus it can be shown that the function

$$
f(x):=(2 x-1)+\sqrt{(2 x-1)^{2}+4(1-x)^{2}}
$$

achieves its minimum on $[-1,1]$ at $x=\frac{1}{2}$. Thus $f(x) \geq f\left(\frac{1}{2}\right)=1$.

Corollary 6.2. For any $0 \leq s \leq r$, we have

$$
s^{2}+2 r s-4 r s \cos \varphi_{1} \leq 4 r^{2}\left(1-\cos \varphi_{1}\right)^{2} .
$$

Proof. It is easy to see that (6.2) is true when

$$
0 \leq s \leq r\left[\left(2 \cos \varphi_{1}-1\right)+\sqrt{\left(2 \cos \varphi_{1}-1\right)^{2}+4\left(1-\cos \varphi_{1}\right)^{2}}\right] .
$$

From Lemma 6.1 we know that

$$
\left(2 \cos \varphi_{1}-1\right)+\sqrt{\left(2 \cos \varphi_{1}-1\right)^{2}+4\left(1-\cos \varphi_{1}\right)^{2}} \geq 1 .
$$

Therefore (6.2) is true for any $0 \leq s \leq r$.

Lemma 6.3. For any $z, w \in \partial D$ with $z \neq w$,

$$
\liminf _{\substack{D \ni x \rightarrow z \\ D \ni y \rightarrow w}} \frac{G_{D}(x, y)}{\delta(x)^{\alpha / 2} \delta(y)^{\alpha / 2}}>0 .
$$

Proof. For any fixed $0<\beta<d_{D}$, the diameter of $D$, let $r=\min \left\{r_{0}, \frac{\beta}{8}\right\}$. For any $z, w \in \partial D$ with $|z-w|>\beta$, when $|x-z|<\frac{r}{2}$ and $|y-w|<\frac{r}{2}$, let $x^{*}$ and $y^{*}$ be the points on $\partial D$ such that $\left|x-x^{*}\right|=\delta(x)$ and $\left|y-y^{*}\right|=\delta(y)$. Write

$$
\begin{array}{ll}
B_{1}^{x^{*}}(r)=B\left(O_{x}, r\right), & B_{1}^{y^{*}}(r)=B\left(O_{y}, r\right) \\
B_{1}^{z}(r)=B\left(O_{z}, r\right), & B_{1}^{w}(r)=B\left(O_{w}, r\right) .
\end{array}
$$

Note that $x \in B\left(O_{x}, r\right)$. By the explicit formula for the Poisson kernel on the ball $B\left(O_{x}, r\right)$ given in [3] and the strong Markov property, we have 


$$
\begin{aligned}
& G_{D}(x, y) \\
& =E_{x}\left[G_{D}\left(X_{\tau_{B\left(O_{x}, r\right)}}, y\right)\right] \\
& =C_{1} \int_{B\left(O_{x}, r\right)^{c} \cap D} \frac{\left(r^{2}-\left|x-O_{x}\right|^{2}\right)^{\alpha / 2}}{\left.|| u-\left.O_{x}\right|^{2}-r^{2}\right)^{\alpha / 2}} \frac{1}{|x-u|^{n}} G_{D}(u, y) d u \\
& \geq C_{1}^{2} \int_{B\left(O_{x}, r\right)^{c} \cap D} \int_{B\left(O_{y}, r\right)^{c} \cap D} \frac{\left(r^{2}-\left|x-O_{x}\right|^{2}\right)^{\alpha / 2}\left(r^{2}-\left|y-O_{y}\right|^{2}\right)^{\alpha / 2}}{\left(\left|u-O_{x}\right|^{2}-r^{2}\right)^{\alpha / 2}\left(\left|v-O_{y}\right|^{2}-r^{2}\right)^{\alpha / 2}} . \\
& \qquad \frac{G_{D}(u, v)}{|x-u|^{n}|y-v|^{n}} d u d v \\
& C_{1}^{2} \int_{D} \int_{D} \frac{\left(r^{2}-\left|x-O_{x}\right|^{2}\right)^{\alpha / 2}\left(r^{2}-\left|y-O_{y}\right|^{2}\right)^{\alpha / 2}}{\left(\left|u-O_{x}\right|^{2}-r^{2}\right)^{\alpha / 2}\left(\left|v-O_{y}\right|^{2}-r^{2}\right)^{\alpha / 2}} \cdot \\
& \quad \cdot \frac{G_{D}(u, v) 1_{B\left(O_{x}, r\right)^{c}}(u) 1_{B\left(O_{y}, r\right)^{c}}(v)}{|x-u|^{n}|y-v|^{n}} d u d v
\end{aligned}
$$

where

$$
C_{1}=\pi^{-\left(\frac{n}{2}+1\right)} \Gamma\left(\frac{n}{2}\right) \sin \frac{\alpha \pi}{2}
$$

Thus

$$
\begin{gathered}
\frac{G_{D}(x, y)}{\delta(x)^{\alpha / 2} \delta(y)^{\alpha / 2}} \geq \quad C_{1}^{2} \int_{D} \int_{D} \frac{\left(r+\left|x-O_{x}\right|\right)^{\alpha / 2}\left(r+\left|y-O_{y}\right|\right)^{\alpha / 2}}{\left(\left|u-O_{x}\right|^{2}-r^{2}\right)^{\alpha / 2}\left(\left|v-O_{y}\right|^{2}-r^{2}\right)^{\alpha / 2}} \\
\quad \cdot \frac{G_{D}(u, v) 1_{B\left(O_{x}, r\right)^{c}}(u) 1_{B\left(O_{y}, r\right)^{c}}(v)}{|x-u|^{n}|y-v|^{n}} d u d v \\
:=h(x, y)
\end{gathered}
$$

We show next that $\lim _{\substack{D \ni x \rightarrow z \\ D \ni y \rightarrow w}} h(x, y)$ exists and forms a positive and continuous function on $\{(z, w) \in \partial D \times \partial D:|z-w|>\beta\}$. For this, we set up a spherical coordinate system $\left(\rho, \varphi_{1}, \ldots, \varphi_{n-1}\right)$ with origin $O_{x}$ and principal axis $\overrightarrow{O_{x} x^{*}}$. Then for any $u=u\left(\rho, \varphi_{1}, \ldots, \varphi_{n-1}\right) \in B\left(O_{x}, r\right)^{c}$, we have

$$
\begin{array}{rll}
|u-x|^{2} & = & \rho^{2}+(r-\delta(x))^{2}-2 \rho(r-\delta(x)) \cos \varphi_{1} \\
\stackrel{\rho=r+s}{=} & 2 r(r+s-\delta(x))\left(1-\cos \varphi_{1}\right)+s^{2}+\delta^{2}(x)+2 s \delta(x) \cos \varphi_{1} \\
& \geq & 4 r(r+s-\delta(x)) \sin ^{2} \frac{\varphi_{1}}{2}+s^{2} \\
& \stackrel{\delta(x)<\frac{r}{2}}{\geq} & 2 r^{2} \sin ^{2} \frac{\varphi_{1}}{2}+s^{2} \\
& \geq\left(2 r^{2} \sin ^{2} \frac{\varphi_{1}}{2}\right) \vee\left(s^{2}\right)
\end{array}
$$

Similarly, if we set up a spherical coordinate system $\left(\gamma ; \theta_{1}, \ldots, \theta_{n-1}\right)$ with origin $O_{y}$ and principal axis $\overrightarrow{O_{y} y^{*}}$, we have for any $v=v\left(\gamma ; \theta_{1}, \ldots, \theta_{n-1}\right) \in B\left(O_{y}, r\right)^{c}$,

$$
|v-y|^{2} \geq\left(2 r^{2} \sin ^{2} \frac{\theta_{1}}{2}\right) \vee\left(t^{2}\right)
$$

where $t=\gamma-r$. Let 


$$
\begin{array}{ll}
D_{1}=\left(B\left(O_{x}, 2 r\right) \backslash B\left(O_{x}, r\right)\right) \cap D, & D_{2}=D \backslash B\left(O_{x}, 2 r\right) \\
D_{3}=\left(B\left(O_{y}, 2 r\right) \backslash B\left(O_{y}, r\right)\right) \cap D, & D_{4}=D \backslash B\left(O_{y}, 2 r\right) .
\end{array}
$$

Then, for any $u \in D_{1}$,

$$
\begin{array}{rll}
d\left(u, \partial B_{2}^{x^{*}}(r)\right) & = & {\left[4 r^{2}+\rho^{2}-4 r \rho \cos \varphi_{1}\right]^{1 / 2}-r} \\
\stackrel{\rho=r+s}{=} & {\left[r^{2}\left(5-4 \cos \varphi_{1}\right)+\left(s^{2}+2 r s-4 r s \cos \varphi_{1}\right)\right]^{1 / 2}-r} \\
\text { Corollary } 6.2 & & {\left[r^{2}\left(5-4 \cos \varphi_{1}\right)+4 r^{2}\left(1-\cos \varphi_{1}\right)^{2}\right]^{1 / 2}-r} \\
& \leq & r\left(\sqrt{5-4 \cos \varphi_{1}}-1\right)+2 r\left(1-\cos \varphi_{1}\right) \\
& \leq & 4 r\left(1-\cos \varphi_{1}\right)+2 r\left(1-\cos \varphi_{1}\right) \\
& = & 12 r \sin ^{2} \frac{\varphi_{1}}{2} .
\end{array}
$$

Similarly, for any $v \in D_{3}$

$$
d\left(v, \partial B_{2}^{y^{*}}(r)\right) \leq 12 r \sin ^{2} \frac{\theta_{1}}{2} .
$$

For $u \in D_{1}, v \in D_{3}$

$$
\begin{aligned}
\beta<|z-w| & \leq|z-x|+|x-u|+|u-v|+|v-y|+|y-w| \\
& \leq \frac{r}{2}+3 r+|u-v|+3 r+\frac{r}{2} \\
& \leq 7 r+|u-v| \\
& \leq \frac{7 \beta}{8}+|u-v|,
\end{aligned}
$$

and hence

$$
|u-v| \geq \frac{\beta}{8}, \quad \forall u \in D_{1}, \forall v \in D_{3} .
$$

By Theorem 1.1, we have

$$
\begin{aligned}
G_{D}(u, v) & \leq C \frac{\delta(u)^{\alpha / 2} \delta(v)^{\alpha / 2}}{|u-v|^{n}} \\
& \leq C\left(\frac{8}{\beta}\right)^{n} d\left(u, \partial B_{2}^{x^{*}}(r)\right)^{\alpha / 2} d\left(v, \partial B_{2}^{y^{*}}(r)\right)^{\alpha / 2} \\
& \leq C(12 r)^{\alpha}\left(\frac{8}{\beta}\right)^{n} \sin ^{\alpha} \frac{\varphi_{1}}{2} \sin ^{\alpha} \frac{\theta_{1}}{2} \quad \text { for }(u, v) \in D_{1} \times D_{3}, \\
G_{D}(u, v) & \leq C \frac{\delta(u)^{\alpha / 2}}{|u-v|^{n-\alpha / 2}} \leq \frac{C(12 r)^{\alpha / 2} \sin ^{\alpha} \frac{\varphi_{1}}{2}}{|u-v|^{n-\alpha / 2}} \quad \text { for }(u, v) \in D_{1} \times D_{4}, \\
G_{D}(u, v) & \leq \frac{C(12 r)^{\alpha / 2} \sin ^{\alpha} \frac{\theta_{1}}{2}}{|u-v|^{n-\alpha / 2}} \quad \text { for }(u, v) \in D_{2} \times D_{3},
\end{aligned}
$$

and

$$
G_{D}(u, v) \leq C \frac{1}{|u-v|^{n-\alpha}} \quad \text { for }(u, v) \in D_{2} \times D_{4}
$$


Thus for $(u, v) \in B\left(O_{x}, r\right)^{c} \times B\left(O_{y}, r\right)^{c}$

$$
\begin{aligned}
& \frac{\left(r+\left|x-O_{x}\right|\right)^{\alpha / 2}\left(r+\left|y-O_{y}\right|\right)^{\alpha / 2}}{\left(\left|u-O_{x}\right|^{2}-r^{2}\right)^{\alpha / 2}\left(\left|v-O_{y}\right|^{2}-r^{2}\right)^{\alpha / 2}} \frac{G_{D}(u, v)}{|u-x|^{n}|v-y|^{n}} \\
& \leq \frac{1}{\left(\left|u-O_{x}\right|-r\right)^{\alpha / 2}\left(\left|v-O_{y}\right|-r\right)^{\alpha / 2}} \text {. } \\
& \left\{\frac{C(12 r)^{\alpha}\left(\frac{8}{\beta}\right)^{n} \sin ^{\alpha} \frac{\varphi_{1}}{2} \sin ^{\alpha} \frac{\theta_{1}}{2} 1_{D_{1}}(u) 1_{D_{3}}(v)}{\left[\left(2 r^{2} \sin ^{2} \frac{\varphi_{1}}{2}\right) \vee\left(\left|u-O_{x}\right|-r\right)^{2}\right]^{n / 2} \cdot\left[\left(2 r^{2} \sin ^{2} \frac{\theta_{1}}{2}\right) \vee\left(\left|v-O_{y}\right|-r\right)^{2}\right]^{n / 2}}\right. \\
& +\frac{C(12 r)^{\alpha / 2} \sin ^{\alpha} \frac{\varphi_{1}}{2} 1_{D_{1}}(u) 1_{D_{4}}(v)}{r^{n}\left[\left(2 r^{2} \sin ^{2} \frac{\varphi_{1}}{2}\right) \vee\left(\left|u-O_{x}\right|-r\right)^{2}\right]^{n / 2}} \frac{1}{|u-v|^{n-\alpha / 2}} \\
& +\frac{C(12 r)^{\alpha / 2} \sin ^{\alpha} \frac{\theta_{1}}{2} 1_{D_{2}}(u) 1_{D_{3}}(v)}{r^{n}\left[\left(2 r^{2} \sin ^{2} \frac{\theta_{1}}{2}\right) \vee\left(\left|v-O_{y}\right|-r\right)^{2}\right]^{n / 2}} \frac{1}{|u-v|^{n-\alpha / 2}} \\
& \left.+\frac{1}{r^{2 n}} \frac{1_{D_{2}}(u) 1_{D_{4}}(v)}{|u-v|^{n-\alpha}}\right\} \\
& \leq C(12 r)^{\alpha}\left(\frac{8}{\beta}\right)^{n} \frac{\sin ^{\alpha} \frac{\varphi_{1}}{2} \cdot 1_{D_{1}}(u)}{\left[\left(2 r^{2} \sin ^{2} \frac{\varphi_{1}}{2}\right) \vee\left(\left|u-O_{x}\right|-r\right)^{2}\right]^{n / 2}} \frac{1}{\left(\left|u-O_{x}\right|-r\right)^{\alpha / 2}} . \\
& \cdot \frac{\sin ^{\alpha} \frac{\theta_{1}}{2} \cdot 1_{D_{3}}(v)}{\left[\left(2 r^{2} \sin ^{2} \frac{\theta_{1}}{2}\right) \vee\left(\left|v-O_{y}\right|-r\right)^{2}\right]^{n / 2}} \frac{1}{\left(\left|v-O_{y}\right|-r\right)^{\alpha / 2}} \\
& +C(12)^{\alpha / 2} r^{-n} \frac{\sin ^{\alpha} \frac{\varphi_{1}}{2} \cdot 1_{D_{1}}(u)}{\left[\left(2 r^{2} \sin ^{2} \frac{\varphi_{1}}{2}\right) \vee\left(\left|u-O_{x}\right|-r\right)^{2}\right]^{n / 2}} \frac{1}{\left(\left|u-O_{x}\right|-r\right)^{\alpha / 2}} \\
& \cdot \frac{1_{D_{4}}(v)}{|u-v|^{n-\alpha / 2}} \\
& +C 12^{\alpha / 2} r^{-n} \frac{\sin ^{\alpha} \frac{\theta_{1}}{2} \cdot 1_{D_{3}}(v)}{\left[\left(2 r^{2} \sin ^{2} \frac{\theta_{1}}{2}\right) \vee\left(\left|v-O_{y}\right|-r\right)^{2}\right]^{n / 2}} \frac{1}{\left(\left|v-O_{y}\right|-r\right)^{\alpha / 2}} \\
& \frac{1_{D_{2}}(u)}{|u-v|^{n-\alpha / 2}} \\
& +C \frac{1}{r^{2 n+\alpha}} \frac{1_{D_{2}}(u) 1_{D_{4}}(v)}{|u-v|^{n-\alpha}} \\
& :=f(u, v ; x, y, z, w) \\
& :=C(12 r)^{\alpha}\left(\frac{8}{\beta}\right)^{n} f_{1}(u, v ; x, y, z, w)+C \cdot 12^{\alpha / 2} r^{-n} f_{2}(u, v ; x, y, z, u) \\
& +C 12^{\alpha} r^{-n} f_{3}(u, v ; x, y, z, w)+C \frac{1}{r^{2 n+\alpha}} f_{4}(u, v ; x, y, z, w) .
\end{aligned}
$$

From Lemma 6.4 below we know that the family of functions of $(u, v)$

$$
\begin{gathered}
\mathscr{b}=\{f(u, v, x, y, z, w): \quad x, y \in D, z, w \in \partial D,|z-w|>\beta, \\
\left.|x-z|<\frac{r}{2},|y-w|<\frac{r}{2}\right\}
\end{gathered}
$$

is uniformly integrable on $D \times D$. 
When $x \rightarrow z, y \rightarrow w$,

$$
\begin{aligned}
\left|O_{x}-O_{z}\right| & =\left|\left(x^{*}+r n_{x^{*}}\right)-\left(z-r n_{z}\right)\right| \\
& \leq\left|x^{*}-z\right|+r C_{0}\left|x^{*}-z\right| \\
& \leq\left(1+r C_{0}\right)\left(\left|x^{*}-x\right|+|x-z|\right) \\
& \leq 2\left(1+r C_{0}\right)|x-z| \rightarrow 0 .
\end{aligned}
$$

Similarly

$$
\left|O_{y}-O_{w}\right| \rightarrow 0 \text {. }
$$

Thus by the uniform integrability of $\mathscr{C}$ in (6.7), we have

$$
\begin{aligned}
& \lim _{\substack{D \ni x \rightarrow z \\
D \ni y \rightarrow w}} h(x, y)= \\
& C_{1}^{2}(2 r)^{\alpha} \int_{D} \int_{D} \frac{G_{D}(u, v) 1_{B\left(O_{z}, r\right)^{c}}(u) 1_{B\left(O_{z}, r\right)^{c}}(v)}{\left(\left|u-O_{z}\right|^{2}-r^{2}\right)^{\alpha / 2}\left(\left|v-O_{w}\right|^{2}-r^{2}\right)^{\alpha / 2}|u-z|^{n}|v-w|^{n}} d u d v \\
& >0
\end{aligned}
$$

and is a continuous function in $\{(z, w) \in \partial D \times \partial D:|z-w|>\beta\}$. Since $\beta>0$ is arbitrary, we have by (6.3) that for any $(z, w) \in \partial D \times \partial D$ with $z \neq w$,

$$
\liminf _{\substack{D \ni x \rightarrow z \\ D \ni y \rightarrow w}} \frac{G_{D}(x, y)}{\delta(x)^{\alpha / 2} \delta(y)^{\alpha / 2}} \geq \lim _{\substack{D \ni x \rightarrow z \\ D \ni y \rightarrow w}} h(x, y)>0 .
$$

Lemma 6.4. There exists $\eta=\eta(n, \alpha)>1$ such that

$$
\sup _{f \in \mathscr{C}} \int_{D} \int_{D}(f(u, v ; x, y, z, w))^{\eta} d u d v<\infty
$$

where. $\mathscr{b}$ is the set of functions defined above in (6.7).

Proof. It is easy to see that for any $1<\eta<\frac{n}{n-\alpha / 2}$, and any $u \in D$

$$
\int_{D} \frac{1}{|u-v|^{(n-\alpha / 2) \eta}} d v \leq \omega_{n} \int_{0}^{d_{D}} \frac{\tau^{n-1}}{\tau^{\eta(n-\alpha / 2)}} d \tau<\infty
$$

and

$$
\int_{D} \int_{D} \frac{1}{|u-v|^{(n-\alpha) \eta}} d u d v \leq|D| \omega_{n} \int_{0}^{d_{D}} \frac{\tau^{n-1}}{\tau^{\eta(n-\alpha)}} d \tau<\infty
$$

Case 1. $1<\alpha<2$.

In this case, we take $1<\eta<\min \left\{\frac{2}{\alpha}, \frac{n-1}{n-\alpha}, \frac{n}{n-\alpha / 2}\right\}$. Then 


$$
\begin{aligned}
& \int_{D}\left[\frac{\sin ^{\alpha} \frac{\varphi_{1}}{2} 1_{D_{1}}(u)}{\left[\left(2 r^{2} \sin ^{2} \frac{\varphi_{1}}{2}\right) \vee\left(\left|u-O_{x}\right|-r\right)^{2}\right]^{n / 2}} \frac{1}{\left(\left|u-O_{x}\right|-r\right)^{\alpha / 2}}\right]^{\eta} d u \\
\leq & \int_{D}\left(\frac{1_{D_{1}}(u)}{2^{n / 2} r^{n} \sin ^{n-\alpha} \frac{\varphi_{1}}{2}} \frac{1}{\left(\left|u-O_{x}\right|-r\right)^{\alpha / 2}}\right) d u \\
\leq & \omega_{n-1} \int_{r}^{2 r} \frac{1}{(\rho-r)^{\alpha \eta / 2}} \int_{0}^{\pi} \frac{1}{\left(2^{n / 2} r^{n} \sin ^{n-\alpha} \frac{\varphi_{1}}{2}\right)^{\eta}} \rho^{n-1} \sin ^{n-2} \varphi_{1} d \varphi_{1} d \rho \\
\leq & \frac{\omega_{n-1}(2 r)^{n-1}}{\left(2^{n / 2} r^{n}\right)^{\eta}} \int_{0}^{r} \frac{1}{s^{\alpha \eta / 2}} d s \int_{0}^{\pi} \frac{\sin ^{n-2} \varphi_{1}}{\sin ^{\eta(n-\alpha)} \frac{\varphi_{1}}{2}} d \varphi_{1}<\infty .
\end{aligned}
$$

Similarly

$$
\begin{aligned}
& \int_{D}\left[\frac{\sin ^{\alpha} \frac{\theta_{1}}{2} 1_{D_{3}}(v)}{\left[\left(2 r^{2} \sin ^{2} \frac{\theta_{1}}{2}\right) \vee\left(\left|v-O_{y}\right|-r\right)^{2}\right]^{n / 2}} \frac{1}{\left(\left|v-O_{y}\right|-r\right)^{\alpha / 2}}\right]^{\eta} d v \\
\leq & \frac{\omega_{n-1}(2 r)^{n-1}}{\left(2^{n / 2} r^{n}\right) \eta} \int_{0}^{r} \frac{1}{s^{\alpha \eta / 2}} d s \int_{0}^{\pi} \frac{\sin ^{n-2} \theta_{1}}{\sin ^{\eta(n-\alpha)} \frac{\theta_{1}}{2}} d \theta_{1}<\infty .
\end{aligned}
$$

Thus, from (6.8)-(6.11), we have

$$
\begin{aligned}
& \int_{D} \int_{D} f_{1}(u, v ; x, y, z, w)^{\eta} d u d v \\
\leq & \frac{\omega_{n-1}^{2}(2 r)^{2 n-2}}{\left(2^{n} r^{2 n}\right)^{\eta}}\left(\int_{0}^{r} s^{-\frac{\alpha \eta}{2}} d s\right)^{2}\left(\int_{0}^{\pi} \frac{\sin ^{n-2} \varphi}{\sin ^{\eta(n-\alpha)} \frac{\varphi}{2}} d \varphi\right)^{2}<\infty, \\
& \int_{D} \int_{D} f_{2}(u, v ; x, y, z, w)^{\eta} d u d v \\
\leq & \frac{\omega_{n} \omega_{n-1}(2 r)^{n-1}}{\left(2^{n / 2} r^{n}\right)^{\eta}}\left(\int_{0}^{r} s^{-\frac{\alpha \eta}{2}} d s\right)\left(\int_{0}^{d_{D}} \frac{\tau^{n-1}}{\tau^{\eta(n-\alpha / 2)}} d \tau\right)\left(\int_{0}^{\pi} \frac{\sin ^{n-\alpha} \varphi}{\sin ^{\eta(n-2)} \frac{\varphi}{2}} d \varphi\right) \\
< & \infty, \quad \int_{D} f_{3}(u, v ; x, y, z, w)^{\eta} d u d v \\
\leq & \frac{\omega_{n} \omega_{n-1}(2 r)^{n-1}}{\left(2^{n / 2} r^{n}\right)^{\eta}}\left(\int_{0}^{r} s^{-\frac{\alpha \eta}{2}} d s\right)\left(\int_{0}^{d_{D}} \frac{\tau^{n-1}}{\tau^{\eta(n-\alpha / 2)}} d \tau\right)\left(\int_{0}^{\pi} \frac{\sin ^{n-2} \varphi}{\sin ^{\eta(n-\alpha)} \frac{\varphi}{2}} d \varphi\right) \\
< & \infty,
\end{aligned}
$$

and

$$
\int_{D} \int_{D} f_{4}(u, v ; x, y, z, w)^{\eta} d u d v \leq|D| \omega_{n}\left(\int_{0}^{d_{D}} \frac{\tau^{n-1}}{\tau^{\eta(n-\alpha)}} d \tau\right)<\infty .
$$

Therefore the assertion of Lemma 6.4 is valid when $1<\alpha<2$.

Case 2. $0<\alpha \leq 1$. 
In this case we take $1<\eta<\min \left\{\frac{n-1}{n-\left(\frac{\alpha}{4}+1\right)}, \frac{4}{3 \alpha}, \frac{n}{n-\frac{\alpha}{2}}\right\}$. Then

$$
\begin{aligned}
& \int_{D}\left[\frac{\sin ^{2} \frac{\varphi_{1}}{2}}{\left[\left(2 r^{2} \sin ^{2} \frac{\varphi_{1}}{2}\right) \vee\left(\left|u-O_{x}\right|-r\right)^{2}\right]^{n / 2}} \frac{1_{D_{1}}(u)}{\left(\left|u-O_{x}\right|-r\right)^{\alpha / 2}}\right]^{\eta} d u \\
\leq & \int_{D}\left[\frac{\sin ^{\alpha} \frac{\varphi_{1}}{2}}{\left(2 r^{2}\right)^{\frac{n-\left(1-\frac{3 \alpha}{4}\right)}{2}} \sin ^{n-\left(1-\frac{3 \alpha}{4}\right)} \frac{\varphi_{1}}{2}} \frac{1_{D_{1}}(u)}{\left(\left|u-O_{x}\right|-r\right)^{1-\frac{\alpha}{4}}}\right]^{\eta} d u \\
\leq & \left(2 r^{2}\right)^{-\frac{n\left(1-\frac{3 \alpha}{4}\right)}{2} \eta} \omega_{n-1} \int_{r}^{2 r} \int_{0}^{\pi} \frac{\sin ^{n-2} \varphi_{1}}{\sin ^{\eta\left(n-\left(1+\frac{\alpha}{4}\right)\right)} \frac{\varphi_{1}}{2}} \frac{1}{(\rho-r)^{\left(1-\frac{\alpha}{4}\right) \eta} \rho^{n-1} d \varphi_{1} d \rho} \\
\leq & \left(2 r^{2}\right)^{-\frac{n-\left(1-\frac{3 \alpha}{4}\right)}{2} \eta}(2 r)^{n-1} \omega_{n-1}\left(\int_{0}^{r} s^{-\left(1-\frac{\alpha}{4}\right) \eta} d s\right) \int_{0}^{\pi} \frac{\sin ^{n-2} \varphi_{1}}{\sin ^{\eta\left(n-\left(1+\frac{\alpha}{4}\right)\right)} \frac{\varphi_{1}}{2}} d \varphi \\
< & \infty .
\end{aligned}
$$

Similarly

$$
\begin{aligned}
& \int_{D}\left[\frac{\sin ^{2} \frac{\theta_{1}}{2} 1_{D_{3}}(v)}{\left[\left(2 r^{2} \sin ^{2} \frac{\theta_{1}}{2}\right) \vee\left(\left|v-O_{y}\right|-r\right)^{2}\right]^{n / 2}} \frac{1}{\left(\left|v-O_{y}\right|-r\right)^{\alpha / 2}}\right]^{\eta} d v \\
\leq & \left(2 r^{2}\right)^{-\frac{n-\left(1-\frac{3 \alpha}{4}\right)}{2} \eta}(2 r)^{n-1} \omega_{n-1}\left(\int_{0}^{r} s^{-\left(1-\frac{\alpha}{4}\right) \eta} d s\right)\left(\int_{0}^{\pi} \frac{\sin ^{n-2} \varphi}{\sin ^{\eta\left(n-\left(1+\frac{\alpha}{4}\right)\right)} \frac{\varphi}{2}} d \varphi\right) \\
< & \infty .
\end{aligned}
$$

Therefore, from (6.8), (6.9), (6.12) and (6.13), we have

$$
\begin{aligned}
& \int_{D} \int_{D} f_{1}(u, v ; x, y, z, w)^{\eta} d u d v \\
\leq & \left(2 r^{2}\right)^{-\left(n-\left(1-\frac{3 \alpha}{4}\right)\right) \eta}(2 r)^{2 n-2} \omega_{n-1}^{2}\left(\int_{0}^{r} s^{-\left(1-\frac{\alpha}{4}\right) \eta} d s\right)^{2} \\
& \quad \times\left(\int_{0}^{\pi} \frac{\sin ^{n-2} \varphi}{\sin ^{\eta\left(n-\left(1+\frac{\alpha}{4}\right)\right)} \frac{\varphi}{2}} d \varphi\right)^{2} \\
< & \infty, \quad \int_{D} f_{2}(u, v ; x, y, z, w)^{\eta} d u d v \\
\leq & \left(2 r^{2}\right)^{-\left(n-\left(1-\frac{3 \alpha}{4}\right)\right) \eta}(2 r)^{n-1} \omega_{n} \omega_{n-1}\left(\int_{0}^{r} s^{-\left(1-\frac{\alpha}{4}\right) \eta} d s\right)\left(\int_{0}^{d_{D}} \frac{\tau^{n-1}}{\tau^{\eta\left(n-\frac{\alpha}{2}\right)}} d \tau\right) \\
& \quad \cdot\left(\int_{0}^{\pi} \frac{\sin ^{n-2} \varphi}{\sin ^{\eta\left(n-\left(1+\frac{\alpha}{4}\right)\right)} \frac{\varphi}{2}} d \varphi\right) \\
<\quad & \infty, \quad
\end{aligned}
$$




$$
\begin{aligned}
& \int_{D} \int_{D} f_{3}(u, v ; x, y, z, w)^{\eta} d u d v \\
\leq \quad & \left(2 r^{2}\right)^{-\frac{n-\left(1-\frac{3 \alpha}{4}\right)}{2} \eta}(2 r)^{n-1} \omega_{n} \omega_{n-1}\left(\int_{0}^{r} s^{-\left(1-\frac{\alpha}{4}\right) \eta} d s\right) \\
& \quad\left(\int_{0}^{d_{D}} \frac{\tau^{n-1}}{\tau^{\eta\left(n-\frac{\alpha}{2}\right)}} d \tau\right)\left(\int_{0}^{\pi} \frac{\sin ^{n-2} \varphi}{\sin ^{\eta\left(n-\left(1+\frac{\alpha}{4}\right)\right)} \frac{\varphi}{2}} d \varphi\right) \\
<\infty & \infty
\end{aligned}
$$

and

$$
\int_{D} \int_{D} f_{4}(u, v ; x, y, z, w)^{\eta} d u d v \leq|D| \omega_{n}(1)\left(\int_{0}^{d_{D}} \frac{\tau^{n-1}}{\tau^{\eta(n-\alpha)}} d \tau\right)<\infty .
$$

Hence the assertion of Lemma 6.4 is also valid when $0<\alpha \leq 1$.

Lemma 6.5. For any $x_{0} \in D$ and $z \in \partial D$, the limit

$$
\lim _{\substack{x \rightarrow x_{0} \\ D \ni y \rightarrow z}} \frac{G_{D}(x, y)}{\delta(y)^{\alpha / 2}}
$$

exists and forms a positive and continuous function on $D \times \partial D$.

Proof. Using the same notations as in the proof of Lemma 6.3, we have

$$
\begin{aligned}
G_{D}(x, y) & =E_{x}\left[G_{D}\left(X_{\tau_{B\left(O_{x}, r\right)}}, y\right)\right] \\
& =C_{1} \int_{B\left(O_{x}, r\right)^{c} \cap D} \frac{\left(r^{2}-\left|x-O_{x}\right|^{2}\right)^{\alpha / 2}}{\left(\left|u-O_{x}\right|^{2}-r^{2}\right)^{\alpha / 2}} \frac{1}{|x-u|^{n}} G_{D}(u, y) d u .
\end{aligned}
$$

From here on we can use a similar but simpler argument as that of Lemma 6.3 to finish the proof of this lemma.

Lemma 6.6. For any ball $B \subset \mathbf{R}^{n}$ of radius $r$, there is a constant $C_{2}=$ $2^{-\alpha} \pi^{-\frac{n}{2}} \Gamma\left(\frac{\alpha}{2}\right)^{-2} \Gamma\left(\frac{n}{2}\right)$ such that

$$
G_{B}(x, y) \geq C_{2}\left(\frac{|x-y|^{n}}{\delta_{B}(x)^{\alpha / 2} \delta_{B}(y)^{\alpha / 2}}+|x-y|^{n-\alpha}\right)^{-1},
$$

where $\delta_{B}(x)=d(x, \partial B)$.

Proof. Without loss of generality, we can assume that $B=B(0,1)$. We know that

$$
G_{B}(x, y)=C_{2} \int_{0}^{z}(u+1)^{-n / 2} u^{\alpha / 2-1} d u|x-y|^{\alpha-n}
$$

where $z=\left(1-|x|^{2}\right)\left(1-|y|^{2}\right)|x-y|^{-2}$. If $z \geq 1$, then

$$
G_{B}(x, y) \geq C_{2} \int_{0}^{1}(u+1)^{-n / 2} u^{\alpha / 2-1} d u|x-y|^{\alpha-n} .
$$


If $z<1$, since

$$
\begin{aligned}
\int_{0}^{z}(u+ & 1)^{-n / 2} u^{\alpha / 2-1} d u=z^{\alpha / 2} \int_{0}^{1}(1+v z)^{-n / 2} v^{\alpha / 2-1} d v \\
& \geq z^{\alpha / 2} \int_{0}^{1}(1+v)^{-n / 2} v^{\alpha / 2-1} d v \\
& \geq(1-|x|)^{\alpha / 2}(1-|y|)^{\alpha / 2}|x-y|^{-\alpha} \int_{0}^{1}(1+v)^{-n / 2} v^{\alpha / 2-1} d v
\end{aligned}
$$

we have

$$
G_{B}(x, y) \geq C_{2} \int_{0}^{1}(1+v)^{-n / 2} v^{\alpha / 2-1} d v \frac{\delta(x)^{\alpha / 2} \delta(y)^{\alpha / 2}}{|x-y|^{n}}
$$

Combining (6.14) and (6.15) we get the assertion of Lemma 6.6.

Proof of Theorem 1.2. If $|x-y| \leq \frac{\delta(x)}{2}$, take $B=B(x, \delta(x))$. Then $y \in B \subset D$. Let $G_{B}$ be the Green function of $B$. Then it follows from [3] that we have

$$
\begin{aligned}
G_{D}(x, y) & \geq G_{B}(x, y) \\
& =c_{1}\left(|x-y|^{\alpha-n}-\int_{|u-x| \geq \delta(x)}|u-x|^{\alpha-n} \mu_{y}(d u)\right) \\
& \geq c_{1}\left(|x-y|^{\alpha-n}-\delta(x)^{\alpha-n}\right) \\
& \geq c_{1}\left(1-2^{\alpha-n}\right) \frac{1}{|x-y|^{n-\alpha}},
\end{aligned}
$$

where $c_{1}=\Gamma\left(\frac{n-\alpha}{2}\right)\left[2^{\alpha} \pi^{n / 2} \Gamma\left(\frac{\alpha}{2}\right)\right]^{-1}$ and $\mu_{y}$ is the distribution of first hitting position of $B(x, \delta(x))^{c}$ when the process starts from $y$.

If $|x-y| \leq \frac{\delta(y)}{2}$, the proof is the same.

In the case that

$$
|x-y|>\max \left\{\frac{\delta(x)}{2}, \frac{\delta(y)}{2}\right\},
$$

if the conclusion of the theorem were false, there must be a sequence $\left\{\left(x_{k}, y_{k}\right)\right\} \subset$ $D \times D$ such that

$$
\left|x_{k}-y_{k}\right|>\max \left\{\frac{\delta\left(x_{k}\right)}{2}, \frac{\delta\left(y_{k}\right)}{2}\right\}, \quad k \geq 1
$$

and

$$
\lim _{k \rightarrow \infty} \frac{G_{D}\left(x_{k}, y_{k}\right)}{\delta\left(x_{k}\right)^{\alpha / 2} \delta\left(y_{k}\right)^{\alpha / 2}}\left|x_{k}-y_{k}\right|^{n}=0 .
$$

By taking subsequence, we may assume $\left(x_{k}, y_{k}\right) \rightarrow(z, w) \in \bar{D} \times \bar{D}$ as $n \rightarrow \infty$. If $z \neq w, z, w \in D$, then

$$
\lim _{k \rightarrow \infty} \frac{G_{D}\left(x_{k}, y_{k}\right)}{\delta\left(x_{k}\right)^{\alpha / 2} \delta\left(y_{k}\right)^{\alpha / 2}}\left|x_{k}-y_{k}\right|^{n}=\frac{G_{D}(z, w)}{\delta(z)^{\alpha / 2} \delta(w)^{\alpha / 2}}|z-w|^{n}>0 .
$$


If $z \neq w, z, w \in \partial D$, then by Lemma 6.3

$$
\liminf _{k \rightarrow \infty} \frac{G_{D}\left(x_{k}, y_{k}\right)}{\delta\left(x_{k}\right)^{\alpha / 2} \delta\left(y_{k}\right)^{\alpha / 2}}\left|x_{k}-y_{k}\right|^{n}=|z-w|^{n} \liminf _{k \rightarrow \infty} \frac{G_{D}\left(x_{k}, y_{k}\right)}{\delta(x)^{\alpha / 2} \delta\left(y_{k}\right)^{\alpha / 2}}>0 .
$$

If $z \neq w, z \in D$ and $w \in \partial D$, then by Lemma 6.5

$$
\lim _{k \rightarrow \infty} \frac{G_{D}\left(x_{k}, y_{k}\right)}{\delta\left(x_{k}\right)^{\alpha / 2} \delta\left(y_{k}\right)^{\alpha / 2}}\left|x_{k}-y_{k}\right|^{n}=\frac{|z-w|^{n}}{\delta(z)^{\alpha / 2}} \lim _{k \rightarrow \infty} \frac{G_{D}\left(x_{k}, y_{k}\right)}{\delta\left(y_{k}\right)^{\alpha / 2}}>0 .
$$

Similarly, if $z \neq w, z \in \partial D$ and $w \in D$, then

$$
\lim _{k \rightarrow \infty} \frac{G_{D}\left(x_{k}, y_{k}\right)}{\delta\left(x_{k}\right)^{\alpha / 2} \delta\left(y_{k}\right)^{\alpha / 2}}\left|x_{k}-y_{k}\right|^{n}>0 .
$$

To deal with the case $z=w\left(x_{k}-y_{k} \rightarrow 0\right)$, we want to prove that there exists a constant $C>0$ such that for any $x$ and $y$ in $D$ satisfying

$$
\max \left\{\frac{\delta(x)}{2}, \frac{\delta(y)}{2}\right\} \leq|x-y|<\frac{r_{0}}{10\left(1+r_{0} C_{0}\right)},
$$

the inequality

$$
G_{D}(x, y) \geq C \frac{\delta(x)^{\alpha / 2} \delta(y)^{\alpha / 2}}{|x-y|^{n}}
$$

holds. This fact leads to the final contradiction.

For any fixed points $x$ and $y$ satisfying (6.16), let $x^{*}$ and $y^{*}$ be the points on $\partial D$ such that $\left|x-x^{*}\right|=\delta(x)$ and $\left|y-y^{*}\right|=\delta(y)$. Set $r=r_{0}, B_{x}=B_{1}^{x^{*}}(r)=$ $B\left(O_{x}, r\right), B_{y}=B_{1}^{y^{*}}(r)=B\left(O_{y}, r\right)$. Then

$$
\begin{aligned}
\left|x^{*}-y^{*}\right| & \leq|x-y|+\delta(x)+\delta(y) \leq 5|x-y| \\
\left|O_{x}-O_{y}\right| & \leq\left|x^{*}-y^{*}\right|+r\left|n_{x^{*}}-n_{y^{*}}\right| \\
& \leq\left(1+r_{0} C_{0}\right)\left|x^{*}-y^{*}\right| \\
& \leq 5\left(1+r_{0} C_{0}\right)|x-y| \leq \frac{r}{2} .
\end{aligned}
$$

Set up a spherical coordinate system $\left(\rho ; \varphi_{1}, \ldots, \varphi_{n-1}\right)$ with origin $O_{x}$ and principal axis $\overrightarrow{O_{x} y}$ (see Fig. 1 below) and set

$$
\begin{aligned}
\theta & =\varphi_{1} \text { coordinate of } O_{y} \\
\bar{\alpha} & =\max \left(\varphi_{1}:\left(r ; \varphi_{1}, \ldots, \varphi_{n-1}\right) \in \bar{B}_{y}\right) \\
S & =\left\{\left(r ; \varphi_{1}, \ldots, \varphi_{n-1}\right) \in \partial B_{x}: 0 \leq \varphi_{1} \leq \bar{\alpha}\right\} \\
I & =\left|y-O_{x}\right| \\
J & =\left|y-O_{y}\right| \\
\beta & =\cos (\theta+\bar{\alpha}) \\
\eta & =\cos \theta .
\end{aligned}
$$


By some elementary geometric arguments, we can get

$$
\begin{aligned}
\left|O_{x}-O_{y}\right| & =2 r \cos (\theta+\bar{\alpha}) \\
\left|y-O_{y}\right|^{2} & =\left|y-O_{x}\right|^{2}+\left|O_{x}-O_{y}\right|^{2}-2\left|y-O_{x}\right|\left|O_{x}-O_{y}\right| \cos \theta .
\end{aligned}
$$

Thus

$$
\begin{aligned}
& I^{2}-4 r \beta \eta I+\left(4 r^{2} \beta^{2}-J^{2}\right)=0 \\
& I=2 r \beta \eta+\left(4 r^{2} \beta^{2} \eta^{2}-4 r^{2} \beta^{2}+J^{2}\right)^{1 / 2} \\
& I-r=\frac{\left(J^{2}-r^{2}\right)+4 r^{2} \beta(\eta-\beta)}{(r-2 r \beta \eta)+\left(4 r^{2} \beta^{2} \eta^{2}-4 r^{2} \beta^{2}+J^{2}\right)^{1 / 2}}
\end{aligned}
$$

Set

$$
\begin{aligned}
h & =16 r \cos (\theta+\bar{\alpha}) \sin \frac{\bar{\alpha}}{2} \sin \left(\theta+\frac{\bar{\alpha}}{2}\right) \\
& =8\left|O_{x}-O_{y}\right| \sin \frac{\bar{\alpha}}{2} \sin \left(\theta+\frac{\bar{\alpha}}{2}\right) .
\end{aligned}
$$

There are three possible cases:

Case 1. $y \notin B_{x}$.

Then $I \geq r$. By (6.19) we get

$$
\begin{aligned}
& r^{2}-J^{2} \leq 4 r^{2} \beta(\eta-\beta)=8 r^{2} \cos (\theta+\bar{\alpha}) \sin \frac{\bar{\alpha}}{2} \sin \left(\theta+\frac{\bar{\alpha}}{2}\right)=\frac{r h}{2}, \\
& \delta(y)=r-J \leq\left(r^{2}-J^{2}\right) / r \leq h .
\end{aligned}
$$

Noting $\beta=\left|O_{x}-O_{y}\right| / 2 r \leq \frac{1}{4}$ and using (6.19), we have

$$
|I-r| \leq \frac{4 r \beta(\eta-\beta)}{1-2 \eta \beta} \leq 8 r \beta(\eta-\beta)=h .
$$

Case 2. $y \in B_{x}$ and $\delta(y) \leq h$.

Then

$$
|I-r|=\delta_{B_{x}}(y) \leq \delta(y) \leq h .
$$

So in both Case 1 and Case 2, the following inequalities

$$
\delta(y) \leq h
$$

$$
|I-r| \leq h
$$

hold.

Since $G_{D}(\cdot, y)$ is superharmonic on $B_{x}$, we have 


$$
\begin{aligned}
G_{D}(x, y) \geq & C_{1} \int_{B_{x}^{c}} \frac{\left(r^{2}-\left|x-O_{x}\right|^{2}\right)^{\alpha / 2}}{\left(\left|u-O_{x}\right|^{2}-r^{2}\right)^{\alpha / 2}} \frac{G_{D}(u, y)}{|u-x|^{n}} d u \\
\geq & C_{1} \int_{B_{x}^{c}} \frac{\left(r^{2}-\left|x-O_{x}\right|^{2}\right)^{\alpha / 2}}{\left(\left|u-O_{x}\right|^{2}-r^{2}\right)^{\alpha / 2}} \frac{G_{B_{y}}(u, y)}{|u-x|^{n}} d u \\
\geq & C_{1} \int_{B_{x}^{c}} \frac{\left(r^{2}-\left|x-O_{x}\right|^{2}\right)^{\alpha / 2}}{\left(\left|u-O_{x}\right|^{2}-r^{2}\right)^{\alpha / 2}} \frac{1_{B_{y}}(u)}{|u-x|^{n}} \cdot \\
& \cdot\left(\frac{|u-y|^{n}}{\delta_{B_{y}}(u)^{\alpha / 2} \delta_{B_{y}}(y)^{\alpha / 2}}+|u-y|^{n-\alpha}\right)^{-1} d u
\end{aligned}
$$

where in the last inequality we used Lemma 6.6.

Put

$$
D_{0}=\left\{u\left(\rho ; \varphi_{1}, \ldots, \varphi_{n-1}\right): 0 \leq \varphi_{1} \leq \frac{\bar{\alpha}}{2}, r \leq \rho \leq r+\frac{h}{24 \pi}\right\},
$$

and let $v$ be the point on $\partial B_{y}$ such that the angle between $\overrightarrow{O_{x} v}$ and $\overrightarrow{O_{x} y}$ is $\frac{\bar{\alpha}}{2}$. (See Fig. 1.)

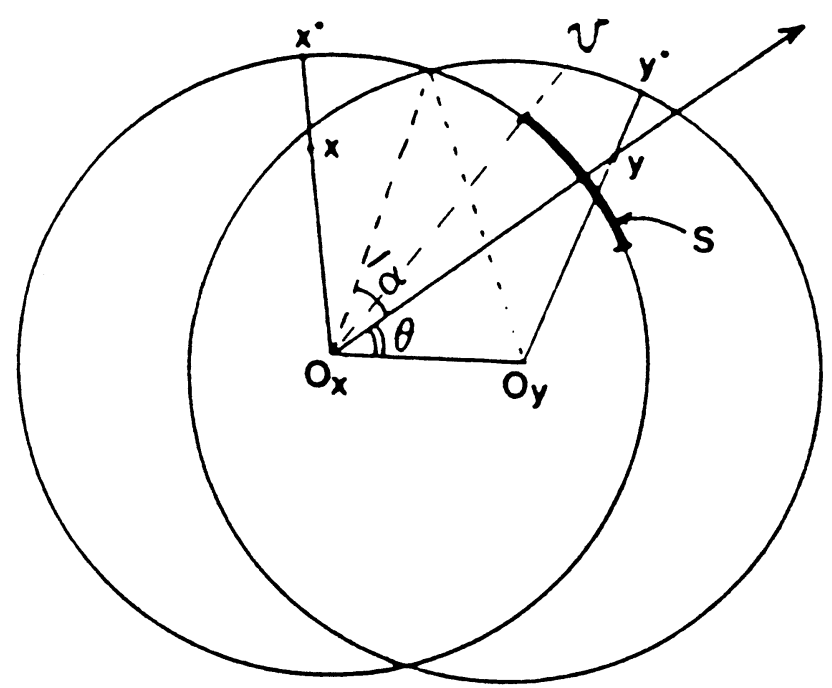

Fig. 1

By the law of cosine we have

$$
\left|v-O_{x}\right|^{2}+\left|O_{x}-O_{y}\right|^{2}-2\left|v-O_{x}\right|\left|O_{x}-O_{y}\right| \cos \left(\theta+\frac{\bar{\alpha}}{2}\right)=r^{2},
$$

i.e.,

$$
\left.\left|v-O_{x}\right|^{2}+4 r^{2} \cos ^{2}(\theta+\bar{\alpha})\right]-4 r\left|v-O_{x}\right| \cos (\theta+\bar{\alpha}) \cos \left(\theta+\frac{\bar{\alpha}}{2}\right)=r^{2}
$$

which can be rewritten as 


$$
\begin{gathered}
\left(\left|v-O_{x}\right|-r\right)^{2}+2\left(\left|v-O_{x}\right|-r\right) r\left(1-2 \cos (\theta+\bar{\alpha}) \cos \left(\theta+\frac{\bar{\alpha}}{2}\right)\right) \\
+4 r^{2} \cos (\theta+\bar{\alpha})\left(\cos (\theta+\bar{\alpha})-\cos \left(\theta+\frac{\bar{\alpha}}{2}\right)\right)=0 .
\end{gathered}
$$

Solving the above equation, we get

$$
\begin{aligned}
& \left|v-O_{x}\right|-r \\
& =-r\left(1-2 \cos (\theta+\bar{\alpha}) \cos \left(\theta+\frac{\bar{\alpha}}{2}\right)\right)+ \\
& +r \sqrt{\left(1-2 \cos (\theta+\bar{\alpha}) \cos \left(\theta+\frac{\bar{\alpha}}{2}\right)\right)^{2}+4 \cos (\theta+\bar{\alpha})\left(\cos \left(\theta+\frac{\bar{\alpha}}{2}\right)-\cos (\theta+\bar{\alpha})\right)} \\
& =\frac{4 r \cos (\theta+\bar{\alpha})\left(\cos \left(\theta+\frac{\bar{\alpha}}{2}\right)-\cos (\theta+\bar{\alpha})\right)}{\left(1-2 \cos (\theta+\bar{\alpha}) \cos \left(\theta+\frac{\bar{\alpha}}{2}\right)\right)+\sqrt{1-4 \cos ^{2}(\theta+\bar{\alpha}) \sin ^{2}\left(\theta+\frac{\bar{\alpha}}{2}\right)}} \\
& \geq 2 r \cos (\theta+\bar{\alpha})\left(\cos \left(\theta+\frac{\bar{\alpha}}{2}\right)-\cos (\theta+\bar{\alpha})\right) \\
& =4 r \cos (\theta+\bar{\alpha}) \sin \left(\theta+\frac{3 \bar{\alpha}}{4}\right) \sin \frac{\bar{\alpha}}{4} \\
& \geq 4 r \cos (\theta+\bar{\alpha}) \sin \left(\theta+\frac{\bar{\alpha}}{2}\right) \sin \frac{\bar{\alpha}}{2} \frac{\sin \frac{\bar{\alpha}}{4}}{\sin \frac{\bar{\alpha}}{2}} \\
& \geq \frac{1}{4 \pi} h, \quad \operatorname{since} \frac{\sin \frac{\bar{\alpha}}{4}}{\sin \frac{\bar{\alpha}}{2}} \geq \frac{1}{\pi} .
\end{aligned}
$$

Therefore $D_{0} \subset B_{y}$, which implies that

$$
\begin{aligned}
G_{D}(x, y) \geq C_{1} \quad & \int_{D_{0}} \frac{\left(r^{2}-\left|x-O_{x}\right|^{2}\right)^{\alpha / 2}}{\left(\left|u-O_{x}\right|^{2}-r^{2}\right)^{\alpha / 2}} \frac{1}{|x-u|^{n}} \\
& \cdot\left(\frac{|u-y|^{n}}{\delta_{B_{y}}(u)^{\alpha / 2} \delta_{B_{y}}(y)^{\alpha / 2}}+|u-y|^{n-\alpha}\right)^{-1} d u .
\end{aligned}
$$

From Zhao [23] we know that for any $\widetilde{u} \in S,|x-\widetilde{u}| \leq 4|x-y|$. For any $u \in D_{0}, \widetilde{u}=\frac{r}{\left|u-O_{x}\right|}\left(u-O_{x}\right)+O_{x} \in S$, thus

$$
\begin{aligned}
|x-u| & \leq|x-\widetilde{u}|+|\widetilde{u}-u| \leq 4|x-y|+\frac{1}{24 \pi} h \\
& \leq 4|x-y|+\frac{1}{24 \pi} 8\left|O_{x}-O_{y}\right| \\
& \leq 4|x-y|+\frac{40}{24 \pi}\left(1+r_{0} C_{0}\right)|x-y| \\
& <5|x-y|,
\end{aligned}
$$

due to the fact that $r_{0} \leq \frac{1}{2 C_{0}}$. Let $\theta_{u}$ be the angle between $\overrightarrow{O_{x} u}$ and $\overrightarrow{O_{x} O_{y}}$. Then $\theta_{u} \leq \theta+\varphi_{1}(u) \leq \theta+\frac{\bar{\alpha}}{2}$. Since 


$$
\begin{array}{cl} 
& \left|u-O_{y}\right|^{2} \\
= & \left|u-O_{x}\right|^{2}+\left|O_{x}-O_{y}\right|^{2}-2\left|u-O_{x}\right|\left|O_{x}-O_{y}\right| \cos \theta_{u} \\
\leq & \left|u-O_{x}\right|^{2}+\left|O_{x}-O_{y}\right|^{2}-2\left|u-O_{x}\right|\left|O_{x}-O_{y}\right| \cos \left(\theta+\frac{\bar{\alpha}}{2}\right) \\
\operatorname{put} \stackrel{\left|u-O_{x}\right|=r+s}{=} & (r+s)^{2}+4 r^{2} \cos ^{2}(\theta+\bar{\alpha})-4 r(r+s) \cos (\theta+\bar{\alpha}) \cos \left(\theta+\frac{\bar{\alpha}}{2}\right),
\end{array}
$$

we have

$$
\begin{aligned}
& r^{2}-\left|u-O_{y}\right|^{2} \\
\geq & 4 r^{2} \cos (\theta+\bar{\alpha})\left(\cos \left(\theta+\frac{\bar{\alpha}}{2}\right)-\cos (\theta+\bar{\alpha})\right) \\
& +4 r s \cos (\theta+\bar{\alpha}) \cos \left(\theta+\frac{\bar{\alpha}}{2}\right)-\left(2 r s+s^{2}\right) \\
\geq \quad & 3 r^{2} \cos (\theta+\bar{\alpha})\left(\cos \left(\theta+\frac{\bar{\alpha}}{2}\right)-\cos (\theta+\bar{\alpha})\right) \\
= & 6 r^{2} \cos (\theta+\bar{\alpha}) \sin \left(\theta+\frac{3 \bar{\alpha}}{4}\right) \sin \frac{\bar{\alpha}}{4} \\
\geq & \frac{6 r^{2}}{\pi} \cos (\theta+\bar{\alpha}) \sin \left(\theta+\frac{\bar{\alpha}}{2}\right) \sin \frac{\bar{\alpha}}{2} \\
= & \frac{3}{8 \pi} r h
\end{aligned}
$$

where in the second inequality from above we used the fact that for $0 \leq s \leq$ $\frac{1}{24 \pi} h$,

$s^{2}+2 r s-4 r s \cos (\theta+\bar{\alpha}) \cos \left(\theta+\frac{\bar{\alpha}}{2}\right) \leq r^{2} \cos (\theta+\bar{\alpha})\left(\cos \left(\theta+\frac{\bar{\alpha}}{2}\right)-\cos (\theta+\bar{\alpha})\right)$

which can be proven by using elementary algebra. Note that

$$
\delta_{B_{y}}(y)=\delta(y)
$$

and

$$
\delta_{B_{y}}(u)=r-\left|u-O_{y}\right| \geq\left(r^{2}-\left|u-O_{y}\right|^{2}\right) / 2 r \geq \frac{3}{16 \pi} h .
$$




$$
\begin{aligned}
& |u-y|^{2} \\
= & \left|y-O_{x}\right|^{2}+\left|u-O_{x}\right|^{2}-2\left|y-O_{x}\right|\left|u-O_{x}\right| \cos \varphi_{1}(u) \\
\left|u-O_{x}\right|=r+s & \rightarrow \\
\rightarrow & I^{2}+(r+s)^{2}-2 I(r+s) \cos \varphi_{1} \\
= & (I-r)^{2}+2 \operatorname{Ir}\left(1-\cos \varphi_{1}\right)+2 r s\left(1-\cos \varphi_{1}\right)+2(r-I) s \cos \varphi_{1}+s^{2} \\
\leq & h^{2}+4 r^{2}\left(1-\cos \varphi_{1}\right)+\frac{r h}{12 \pi}\left(1-\cos \varphi_{1}\right)+\frac{h^{2}}{12 \pi}+\frac{h^{2}}{(24 \pi)^{2}}, \\
\leq & \left(\frac{3 h}{2}\right)^{2}+5 r^{2}\left(1-\cos \varphi_{1}\right) \\
\leq & \left(\frac{3}{2} h\right)^{2}+5 r^{2} \sin ^{2} \varphi_{1} .
\end{aligned}
$$

From (6.20)-(6.26), we have

$$
\begin{aligned}
& G_{D}(x, y) \\
& \geq C_{1} \frac{\left(r^{2}-\left|x-O_{x}\right|^{2}\right)^{\alpha / 2}}{5^{n}|x-y|^{n}} \int_{D_{0}} \frac{1}{\left(\left|u-O_{x}\right|^{2}-r^{2}\right)^{\alpha / 2}} \cdot \\
& \cdot\left(\left(\frac{16 \pi}{3}\right)^{\alpha / 2} \frac{1}{h^{\alpha / 2} \delta(y)^{\alpha / 2}}\left[\left(\frac{3}{2} h\right)^{2}+5 r^{2} \sin ^{2} \varphi_{1}\right]^{n / 2}\right. \\
&\left.+\left[\left(\frac{3}{2} h\right)^{2}+5 r^{2} \sin ^{2} \varphi_{1}\right]^{n / 2-\alpha / 2}\right)^{-1} d u \\
& \geq \frac{C_{1} \delta(x)^{\alpha / 2}}{3^{\alpha / 2} 5^{n}|x-y|^{n}} \int_{D_{0}} \frac{1}{\left(\left|u-O_{x}\right|-r\right)^{\alpha / 2}} \cdot\left(\left(\frac{16 \pi}{3}\right)^{\alpha / 2} \frac{1}{h^{\alpha / 2} \delta(y)^{\alpha / 2}} \cdot\right. \\
& \\
&\left.\quad \cdot\left[\left(\frac{3 h}{2}\right)^{2}+5 r^{2} \sin ^{2} \varphi_{1}\right]^{n / 2}+\left[\left(\frac{3 h}{2}\right)^{2}+5 r^{2} \sin ^{2} \varphi_{1}\right]^{n / 2-\alpha / 2}\right)^{-1} d u \\
&=\frac{C_{1} \omega_{n-1}}{3^{\alpha / 2} 5^{n}} \frac{\delta(x)^{\alpha / 2}}{|x-y|^{n}} \int_{r}^{r+\frac{h}{24 \pi}} \int_{0}^{\frac{\alpha}{2}} \frac{1}{(\rho-r)^{\alpha / 2}} \rho^{n-1} \sin ^{n-2} \varphi_{1} \\
& \cdot\left(\left(\frac{16 \pi}{3}\right)^{\alpha / 2} \frac{1}{h^{\alpha / 2} \delta(y)^{\alpha / 2}}\left[\left(\frac{3}{2} h\right)^{2}+5 r^{2} \sin ^{2} \varphi_{1}\right]^{n / 2}\right. \\
&\left.+\left[\left(\frac{3}{2} h\right)^{2}+5 r^{2} \sin ^{2} \varphi_{1}\right]^{n / 2-\alpha / 2}\right)^{-1} d \varphi_{1} d \varrho \\
& \geq \frac{C_{1} \omega_{n-1}}{3^{\alpha / 2} 5^{n}} \frac{\delta(x)^{\alpha / 2}}{|x-y|^{n}} \int_{0}^{\frac{h}{24 \pi}} s^{-\alpha / 2} d s \int_{0}^{\frac{\alpha}{2}} r^{n-1} \sin ^{n-2} \varphi_{1} \\
& \cdot\left(\left(\frac{16 \pi}{3}\right)^{\alpha / 2} \frac{1}{h^{\alpha / 2} \delta(y)^{\alpha / 2}}\left[\left(\frac{3}{2} h\right)^{2}+5 r^{2} \sin ^{2} \varphi_{1}\right]^{n / 2}\right. \\
&+ {\left.\left[\left(\frac{3}{2} h\right)^{2}+5 r^{2} \sin ^{2} \varphi_{1}\right]^{n / 2-\alpha / 2}\right)^{-1} d \varphi_{1} . }
\end{aligned}
$$


Note that

$$
\begin{aligned}
& \int_{0}^{\frac{\bar{\alpha}}{2}}\left(\left(\frac{16 \pi}{3}\right)^{\alpha / 2} \frac{1}{h^{\alpha / 2} \delta(y)^{\alpha / 2}}\left[\left(\frac{3}{2} h\right)^{2}+5 r^{2} \sin ^{2} \varphi_{1}\right]^{n / 2}\right. \\
& \left.+\left[\left(\frac{3}{2} h\right)^{2}+5 r^{2} \sin ^{2} \varphi_{1}\right]^{n / 2-\alpha / 2}\right)^{-1} r^{n-1} \sin ^{n-2} \varphi_{1} d \varphi_{1} \\
\geq & \int_{0}^{r \sin \frac{\bar{\alpha}}{2}}\left(\left(\frac{16 \pi}{3}\right)^{\alpha / 2} \frac{1}{h^{\alpha / 2} \delta(y)^{\alpha / 2}}\left[\left(\frac{3}{2} h\right)^{2}+5 v^{2}\right]^{n / 2}+\right. \\
& \left.+\left[\left(\frac{3}{2} h\right)+5 v^{2}\right]^{n / 2-\alpha / 2}\right)^{-1} v^{n-2} d v \\
= & \int_{0}^{\frac{2 r \sin \frac{\alpha}{2}}{3 h}}\left(\frac{3}{2} h\right)^{\alpha-1}\left(\left(\frac{16 \pi}{3}\right)^{\alpha / 2}\left(\frac{3}{2}\right)^{\alpha} \frac{h^{\alpha / 2}}{\delta(y)^{\alpha / 2}}\left(1+5 w^{2}\right)^{n / 2}+\right. \\
& \left.+\left(1+5 w^{2}\right)^{n / 2-\alpha / 2}\right)^{-1} w^{n-2} d w \\
= & \delta(y)^{\alpha / 2}\left(\frac{3}{2} h\right)^{\alpha / 2-1}\left(\frac{3}{2}\right)^{\alpha / 2} \int_{0}^{\frac{2 r \sin \frac{\alpha}{2}}{3 h}} \frac{h^{\alpha / 2}}{\delta(y)^{\alpha / 2}} \cdot \\
& +\left(\left(\frac{16 \pi}{3}\right)^{\alpha / 2}\left(\frac{3}{2}\right)^{\alpha} \frac{h^{\alpha / 2}}{\delta(y)^{\alpha / 2}}\left(1+5 w^{2}\right)^{n / 2}+\left(1+5 w^{2}\right)^{n / 2-\alpha / 2}\right)^{-1} w^{n-2} d w \\
\geq & \delta(y)^{\alpha / 2}\left(\frac{3}{2} h\right)^{\alpha / 2-1}\left(\frac{3}{2}\right)^{\alpha / 2} \int_{0}^{1 / 6}\left(\left(\frac{16 \pi}{3}\right)^{\alpha / 2}\left(\frac{3}{2}\right)^{\alpha}\left(1+5 w^{2}\right)^{n / 2}+\right. \\
& \left.+\left(1+5 w^{2}\right)^{n / 2-\alpha / 2}\right)^{-1} w^{n-2} d w \\
= & \delta(y)^{\alpha / 2} h^{\alpha / 2-1} \cdot
\end{aligned}
$$

Therefore

$$
\begin{gathered}
G_{D}(x, y) \geq C \frac{\delta(x)^{\alpha / 2} \delta(y)^{\alpha / 2}}{|x-y|^{n}} h^{\alpha / 2-1} \int_{0}^{\frac{h}{24 \pi}} s^{-\alpha / 2} d s \\
=C \frac{\delta(x)^{\alpha / 2} \delta(y)^{\alpha / 2}}{|x-y|^{n}} .
\end{gathered}
$$

Case 3. $y \in B_{x}, \delta(y)>h$.

Since $I<r$ and $(r-2 r \beta \eta)+\left(4 r^{2} \beta^{2} \eta^{2}-4 r^{2} \beta^{2}+J^{2}\right)^{1 / 2} \leq 2 r$. We have from (6.19) that

$$
\begin{aligned}
\delta_{B_{x}}(y)=r-I & \geq \frac{\left(r^{2}-J^{2}\right)-4 r^{2} \beta(\eta-\beta)}{2 r} \\
& \geq \frac{1}{2}[(r-J)-4 r \beta(\eta-\beta)] \\
& =\frac{1}{2}\left[\delta(y)-\frac{h}{2}\right] .
\end{aligned}
$$

Thus 


$$
\begin{aligned}
& \delta(y) \leq 2 \delta_{B_{x}}(y)+\frac{h}{2} \leq 2 \delta_{B_{x}}(y)+\frac{\delta(y)}{2} \\
& \delta(y) \leq 4 \delta_{B_{x}}(y) \\
& \delta(x)=\delta_{B_{x}}(x) .
\end{aligned}
$$

Therefore by Lemma 6.6 we have

$$
\begin{aligned}
G_{D}(x, y) & \geq G_{B_{x}}(x, y) \\
& \geq C_{2}\left(\frac{|x-y|^{n}}{\delta_{B_{x}}(x)^{\alpha / 2} \delta_{B_{x}}(y)^{\alpha / 2}}+|x-y|^{n-\alpha}\right)^{-1} \\
& \geq C_{2}\left(\frac{4^{\alpha / 2}|x-y|^{n}}{\delta(x)^{\alpha / 2} \delta(y)^{\alpha / 2}}+|x-y|^{n-\alpha}\right)^{-1} \\
& \geq C_{2}\left(\frac{4^{\alpha / 2}|x-y|^{n}}{\delta(x)^{\alpha / 2} \delta(y)^{\alpha / 2}}+\frac{4^{\alpha / 2}|x-y|^{\alpha}}{\delta(x)^{\alpha / 2} \delta(y)^{\alpha / 2}}|x-y|^{n-\alpha}\right)^{-1} \\
& =\frac{C_{2}}{2^{\alpha+1}} \frac{\delta(x)^{\alpha / 2} \delta(y)^{\alpha / 2}}{|x-y|^{n}} .
\end{aligned}
$$

Inequality (6.17) follows from (6.27) and (6.28). The proof is now completed.

\section{References}

1. J. Bertoin: Lévy Processes, Cambridge University Press, 1996.

2. R. M. Blumenthal, R. K. Getoor: Markov Processes and Potential Theory, Academic Press, 1968.

3. R. M. Blumenthal, R. K. Getoor, D. B. Ray: On the distribution of first hits for the symmetric stable processes, Trans. Amer. Math. Soc. 99 (1961), 540-554.

4. K. Bogdan: The boundary Harnack principle for the fractional Laplacian, Studia Math. 123(1) (1997), 43-80.

5. Z. Q. Chen, R. Song: Intrinsic ultracontractivity and conditional gauge for symmetric stable processes, J. Funct. Analysis 150 (1997), 204-239.

6. K. L. Chung, Z. Zhao: From Brownian motion to Schrödinger's equation (1995), Springer, Berlin

7. M. Cranston: Lifetime of conditional Brownian motion in Lipschitz domains, Z. Wahrsch. Verw. Gebiete. 70 (1985), 335-340.

8. M. Cranston, E. Fabes, Z. Zhao: Conditional gauge and potential theory for the Schrödinger operator., Trans. Amer. Math. Soc. 307 (1988), 174-194.

9. J. Elliott: Dirichlet spaces associated with integro-differential operators, Part I, Ill. J. Math. 9 (1965), 87-98.

10. J. Elliott: Dirichlet spaces associated with integro-differential operators, Part II Ill. J. Math. 10 (1966), 66-89.

11. M. Fukushima, Y. Oshima, M. Takeda: Dirichlet forms and Symmetric Markov Processes, Walter de Gruyter, 1994.

12. R. Getoor: First passage times for symmetric stable processes in space Trans. Amer. Math. Soc. 101 (1961), 75-90.

13. N. Ikeda, S. Watanabe: On some relations between the harmonic measure and the Lévy measure for a certain class of Markov processes J. Math. Kyoto Univ. 2-1 (1962), 79-95.

14. A. Janicki, A. Weron: Simulation and Chaotic Behavior of $\alpha$-Stable Processes, Dekker, 1994.

15. J. Klafter, M. F. Shlesinger, G. Zumofen: Beyond Brownian motion., Physics Today 49 No. 2 (1996), 33-39.

16. N. S. Landkof: Foundations of Modern Potential Theory, Springer-Verlag, 1970.

17. H. P. McKean: Sample functions of stable processes, Ann. Math. 61 (1955), 564-579.

18. P. W. Millar: First passage distributions of processes with independent increments, Ann. Prob. 3 (1975), 215-233. 
19. M. Sharpe: General theory of Markov processes, Academic Press, 1988.

20. M. L. Silverstein: Symmetric Markov Processes, Springer-Verlag, 1974.

21. M. L. Silverstein: Boundary Theory for Symmetric Markov Processes, Springer-Verlag, 1976.

22. Z. Zhao: Uniform boundedness of conditional gauge and Schrödinger equations, Commun. Math. Phys. 93 (1984), 19-31.

23. Z. Zhao: Green function for Schrödinger operator and conditioned Feynman-Kac gauge, J. Math. Anal. Appl. 116 (1986), 309-334.

Note added in proof. The first version of this paper was finished in September of 1996. The results of this paper were presented by the authors at the probability seminars of Cornell University, Duke University, New York University in October of 1996. After this paper was accepted, we found out that part of the results of this paper were independently obtained by T. Kulczcki in the paper "Properties of Green functions of symmetric stable processes", Probability and Mathematical Statistics, Vol. 17, 1997, pp. 339-364. We thank K. Bogdan for bringing this paper to our attention. 